\title{
Vibration Analysis of Piezoelectric Composite Plate Resting on Nonlinear Elastic Foundations Using Sinc and Discrete Singular Convolution Differential Quadrature Techniques
}

\author{
Ola Ragb, Mohamed Salah $(\mathbb{D}$, M. S. Matbuly, and R. B. M. Amer \\ Department of Engineering Mathematics and Physics, Faculty of Engineering, Zagazig University, 44519 Zagazig, Egypt \\ Correspondence should be addressed to Mohamed Salah; m_2010_salah@yahoo.com
}

Received 10 April 2020; Accepted 25 May 2020; Published 15 July 2020

Academic Editor: Pietro Bia

Copyright (c) 2020 Ola Ragb et al. This is an open access article distributed under the Creative Commons Attribution License, which permits unrestricted use, distribution, and reproduction in any medium, provided the original work is properly cited.

\begin{abstract}
In this work, free vibration of the piezoelectric composite plate resting on nonlinear elastic foundations is examined. The threedimensionality of elasticity theory and piezoelectricity is used to derive the governing equation of motion. By implementing two differential quadrature schemes and applying different boundary conditions, the problem is converted to a nonlinear eigenvalue problem. The perturbation method and iterative quadrature formula are used to solve the obtained equation. Numerical analysis of the proposed schemes is introduced to demonstrate the accuracy and efficiency of the obtained results. The obtained results are compared with available results in the literature, showing excellent agreement. Additionally, the proposed schemes have higher efficiency than previous schemes. Furthermore, a parametric study is introduced to investigate the effect of elastic foundation parameters, different materials of sensors and actuators, and elastic and geometric characteristics of the composite plate on the natural frequencies and mode shapes.
\end{abstract}

\section{Introduction}

The increase in the use of piezoelectric composite materials resting on elastic foundation structures, especially in aerospace, automotive, and marine environments, leads to the rise of difficulties in nonlinear vibrations in various modern engineering challenges. The elastic foundations play an important role that preserve the structural system under oscillations and avoid mechanical failures. The main role of nonlinear foundations is developing the accuracy of the model to describe the behavior of our system. The cubic nonlinearity is the most common type used in recent research [1-8].

Due to the mathematical complexity of such problems, only limited cases can analytically be solved. A number of exact methods of the vibration problems on elastic foundations are issued in [9-14]. Literature on the numerical solution of the research subject is sparse. Typical useful numerical methods, such as the finite element [15-17], the boundary element [18], differential quadrature [19], and state space $[20,21]$ techniques, are used to solve such problems. One of the disadvantages of these methods is consuming too much time and effort to get the solution [22-26]. Additionally, there is no research in piezoelectric composite plate resting on nonlinear elastic foundations, whether analytically or numerically.

The sinc differential quadrature method (SDQM) [27-32] and the discrete singular convolution differential quadrature method (DSCDQM) [33-42] are more reliable than the polynomial-based DQM. The nonlinear partial differential equations are reduced to linear equations using perturbation [43] and iterative quadrature methods [44]. Previous DQ techniques are then employed to reduce the problem to the eigenvalue or bending problem. The same schemes are used for free vibration analysis of piezoelectric nanobeams $[45,46]$. Recent studies have investigated the vibration analysis of composite plate resting on elastic foundations, using DSCDQM [47-58].

As far as the authors are aware, SDQM and DSCDQM have not been examined for vibration analysis of composite piezoelectric plate materials resting on nonlinear elastic foundations. Based on these versions, numerical schemes are 
designed for free vibration of piezoelectric composites. The natural frequencies are obtained and compared with previous analytical and numerical frequencies. For each scheme, the convergence and efficiency are verified. Additionally, a parametric study is introduced to investigate the influence of elastic foundation parameters and elastic and geometric characteristics of the composite on the vibrated results.

\section{Formulation of the Problem}

Consider a three-dimensional piezoelectric composite with $(0 \leq x \leq a, 0 \leq y \leq b, 0 \leq z \leq h)$, where $a, b$, and $h$ are the length, width, and total thickness of the composite, respectively. This composite is polarized in the $z$ direction and consists of $m$ layers with different types of materials. The plate rests on a three-parameter foundation model, as shown in Figure 1.

Based on the theory of elasticity and piezoelectricity, the equations of motion and the charge equation of electrostatic can be written as follows [59, 65-69]:

$$
\begin{gathered}
\sigma_{x, x}+\tau_{x y, y}+\tau_{x z, z}=\rho u_{, t t}, \\
\tau_{x y, x}+\sigma_{y, y}+\tau_{y z, z}=\rho v_{, t t}, \\
\tau_{x z, x}+\tau_{y z, y}+\sigma_{z, z}+k_{1} w+k_{3} w^{3}-k_{2}\left(w_{, x x}+w_{, y y}\right)=\rho w_{, t t},
\end{gathered}
$$

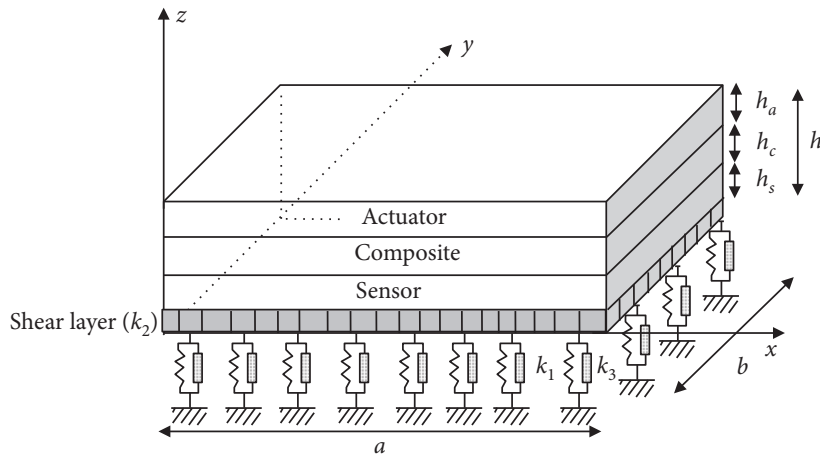

FIgUre 1: Piezoelectric composite (sensor is PZT-4 and actuator is $\mathrm{Ba}_{2} \mathrm{NaNb}_{5} \mathrm{O}_{15}$ ) resting on nonlinear elastic foundations.

$$
D_{x, x}+D_{y, y}+D_{z, z}=0
$$

where $\left(\sigma_{x}, \sigma_{y}, \sigma_{z}\right),(u, v, w)$, and $\left(D_{x}, D_{y}, D_{z}\right)$ are the stresses, displacement, and induction field in the $x, y$, and $z$ directions, respectively; $\left(\tau_{y z}, \tau_{x z}, \tau_{x y}\right)$ are the shear stresses; $\rho$ is the density of the material; and $\left(k_{1}, k_{2}, k_{3}\right)$ are linear Winkler foundation, linear Pasternak foundation, and nonlinear Winkler foundation parameters, respectively.

The relation between mechanical and electric material properties is constitutive equations, which can be written as follows:

$$
\begin{aligned}
& \left.\left(\begin{array}{c}
\sigma_{x} \\
\sigma_{y} \\
\sigma_{z} \\
\tau_{y z} \\
\tau_{x z} \\
\tau_{x y}
\end{array}\right)=\left[\begin{array}{cccccc}
C_{11} & C_{12} & C_{13} & 0 & 0 & 0 \\
C_{12} & C_{11} & C_{13} & 0 & 0 & 0 \\
C_{13} & C_{13} & C_{33} & 0 & 0 & 0 \\
0 & 0 & 0 & C_{44} & 0 & 0 \\
0 & 0 & 0 & 0 & C_{55} & 0 \\
0 & 0 & 0 & 0 & 0 & C_{66}
\end{array}\right]\left(\begin{array}{c}
u_{, x} \\
v_{, y} \\
w_{, z} \\
w_{, y}+v_{, z} \\
w_{, x}+u_{, z} \\
v_{, x}+u_{, y}
\end{array}\right)+\left[\begin{array}{llllll}
0 & 0 & 0 & 0 & e_{5} & 0 \\
0 & 0 & 0 & e_{4} & 0 & 0 \\
0 & 0 & e_{3} & 0 & 0 & 0
\end{array}\right]\right]^{T}\left(\begin{array}{l}
\phi_{, x} \\
\phi_{, y} \\
\phi_{, z}
\end{array}\right), \\
& \left(\begin{array}{c}
D_{x} \\
D_{y} \\
D_{z}
\end{array}\right)=\left[\begin{array}{llllll}
0 & 0 & 0 & 0 & e_{5} & 0 \\
0 & 0 & 0 & e_{4} & 0 & 0 \\
0 & 0 & e_{3} & 0 & 0 & 0
\end{array}\right]\left(\begin{array}{c}
u_{, x} \\
v_{, y} \\
w_{, z} \\
w_{, y}+v_{, z} \\
w_{, x}+u_{, z} \\
v_{, x}+u_{, y}
\end{array}\right)-\left[\begin{array}{ccc}
\eta_{1} & 0 & 0 \\
0 & \eta_{2} & 0 \\
0 & 0 & \eta_{3}
\end{array}\right]\left(\begin{array}{l}
\phi_{, x} \\
\phi_{, y} \\
\phi_{, z}
\end{array}\right),
\end{aligned}
$$

where $C, e$, and $\eta$ are the components of the effective elastic, piezoelectric, and dielectric constants of the same piezoelectric material, respectively, and $\phi$ is the electric potential.

For harmonic behavior, one can assume that

$$
\begin{aligned}
u(x, z, t) & =U e^{i \omega t}, \\
v(x, z, t) & =V e^{i \omega t}, \\
w(x, z, t) & =W e^{i \omega t}, \\
\phi(x, z, t) & =\Phi e^{i \omega t},
\end{aligned}
$$


where $\omega$ is the natural frequency of the plate and $i=\sqrt{-1}$. $U, W$, and $\Phi$ are the amplitudes for $u, w$, and $\phi$, respectively.
The elastic material constants can be determined as follows, using the reciprocal theorem [41]:

$$
\begin{aligned}
& C_{11}=\frac{E_{1}\left(E_{2}-v_{23}^{2} E_{3}\right)}{E_{2}-v_{12}^{2} E_{2}^{2} / E_{1}-v_{23}^{2} E_{3}-v_{13} E_{2} E_{3} / E_{1}\left(v_{13}-2 v_{12} v_{23}\right)}, \\
& C_{12}=C_{21}=\frac{v_{12} E_{2}+v_{13} v_{23} E_{3}}{1-v_{12}^{2} E_{2} / E_{1}-v_{23}^{2} E_{3} / E_{2}-v_{13} E_{3} / E_{1}\left(v_{13}-2 v_{12} v_{23}\right)}, \\
& C_{13}=C_{31}=\frac{E_{3}\left(v_{13}+v_{12} v_{23}\right)}{1-v_{12}^{2} E_{2} / E_{1}-v_{23}^{2} E_{3} / E_{2}-v_{13} E_{3} / E_{1}\left(v_{13}-2 v_{12} v_{23}\right)}, \\
& C_{22}=\frac{E_{2}\left(E_{1}-v_{13}^{2} E_{3}\right)}{E_{1}-v_{12}^{2} E_{2}-v_{23}^{2} E_{1} E_{3} / E_{2}-v_{13} E_{3}\left(v_{13}-2 v_{12} v_{23}\right)}, \\
& C_{23}=C_{32}=\frac{E_{3}\left(v_{23} E_{1}+v_{12} v_{13} E_{2}\right)}{E_{1}-v_{12}^{2} E_{2}-v_{23}^{2} E_{1} E_{3} / E_{2}-v_{13} E_{3}\left(v_{13}-2 v_{12} v_{23}\right)}, \\
& C_{33}=\frac{E_{3}\left(E_{1}-v_{12}^{2} E_{2}\right)}{E_{1}-v_{12}^{2} E_{2}-v_{23}^{2} E_{1} E_{3} / E_{2}-v_{13} E_{3}\left(v_{13}-2 v_{12} v_{23}\right)}, \\
& C_{44}=G_{23}, \\
& C_{55}=G_{13}, \\
& C_{66}=G_{12},
\end{aligned}
$$

where $E_{p}, G_{p q}$, and $v_{p q}(p, q=1,2,3)$ are Young's moduli, shear moduli, and Poisson's ratios.
The isotropic material constants can be expressed as follows [56]:

$$
\begin{aligned}
& C_{11}=C_{12}=C_{13}=C_{22}=C_{23}=C_{31}=C_{32}=C_{33}=\frac{\nu \mathrm{E}}{(1+\nu)(1-2 \nu)}+2 \frac{\mathrm{E}}{2(1+\nu)}, \\
& C_{44}=C_{55}=C_{66}=\frac{\mathrm{E}}{2(1+\nu)} .
\end{aligned}
$$

Substituting equations (5)-(16) into (1)-(4), the problem can be reduced to a quasistatic one, as follows:

$$
\begin{gathered}
C_{11} U_{, x x}+C_{66} U_{, y y}+C_{55} U_{, z z}+\left(C_{12}+C_{66}\right) V_{, x y}+\left(C_{13}+C_{55}\right) W_{, x z}+\left(e_{1}+e_{5}\right) \Phi_{, x z}=-\rho \omega^{2} U \\
\left(C_{66}+C_{12}\right) U_{, x y}+C_{66}^{2} V_{, x x}+C_{11} V_{, y y}+C_{44} V_{, z z}+\left(C_{13}+C_{44}\right) W_{, y z}+\left(e_{2}+e_{4}\right) \Phi_{, y z}=-\rho \omega^{2} V, \\
\left(C_{55}+C_{13}\right) U_{, x z}+\left(C_{13}+C_{44}\right) V_{, y z}+\left(C_{55}-k_{2}\right) W_{, x x}+\left(C_{44}-k_{2}\right) W_{, y y} \\
\quad+C_{33} W_{, z z}+e_{5} \Phi_{, x x}+e_{4} \Phi_{, y y}+e_{3} \Phi_{, z z}+k_{1} W+k_{3} W^{3}=-\rho \omega^{2} W \\
\left(e_{1}+e_{5}\right) U_{, x z}+\left(e_{2}+e_{4}\right) V_{, y z}+e_{5} W_{, x x}+e_{4} W_{, y y}+e_{3} W_{, z z}-\eta_{1} \Phi_{, x x}-\eta_{2} \Phi_{, y y}-\eta_{3} \Phi_{, z z}=0 .
\end{gathered}
$$


The boundary conditions can be described as follows:

(1) For a simply supported edge (S):

$$
\begin{aligned}
& w=v=\sigma_{x}=0, \text { at } x=0, a, \\
& w=u=\sigma_{y}=0, \text { at } y=0, b .
\end{aligned}
$$

(2) For a clamped edge (C):

$$
w=v=u=0, \text { at } x=0, a y=0, b .
$$

(3) For a free edge (F):

$$
\begin{gathered}
\sigma_{x}=\tau_{x z}=\tau_{x y}=0, \text { at } x=0, a, \\
\sigma_{y}=\tau_{y z}=\tau_{x y}=0, \text { at } y=0, b .
\end{gathered}
$$

Mechanical and electrical boundary conditions at the lower and upper surfaces of the composite are as follows:

$$
\begin{gathered}
\sigma_{z}=\tau_{x z}=\tau_{z y}=D_{z}=0, \text { at } z=0, \\
\sigma_{z}=\tau_{x z}=\tau_{z y}=\Phi=0, \text { at } z=h,
\end{gathered}
$$

To ensure the continuity between electric and elastic layers, the following conditions can be considered:

$$
\begin{gathered}
U\left(x, y, h_{s}^{-}\right)=U\left(x, y, h_{s}^{+}\right), \\
V\left(x, y, h_{s}^{-}\right)=V\left(x, y, h_{s}^{+}\right) \\
W\left(x, y, h_{s}^{-}\right)=W\left(x, y, h_{s}^{+}\right), \\
\Phi\left(x, y, h_{s}^{-}\right)=\Phi\left(x, y, h_{s}^{+}\right),
\end{gathered}
$$

$$
\begin{aligned}
U\left(x, y,\left(h_{s}+h_{c}\right)^{-}\right) & =U\left(x, y,\left(h_{s}+h_{c}\right)^{+}\right), \\
V\left(x, y,\left(h_{s}+h_{c}\right)^{-}\right) & =V\left(x, y,\left(h_{s}+h_{c}\right)^{+}\right), \\
W\left(x, y,\left(h_{s}+h_{c}\right)^{-}\right) & =W\left(x, y,\left(h_{s}+h_{c}\right)^{+}\right), \\
\Phi\left(x, y,\left(h_{s}+h_{c}\right)^{-}\right) & =\Phi\left(x, y,\left(h_{s}+h_{c}\right)^{+}\right) .
\end{aligned}
$$

Additionally, the continuity conditions between different elastic materials are as follows:

$$
\begin{gathered}
U\left(x, y, h_{k}^{-}\right)=U\left(x, y, h_{k}^{+}\right), \\
V\left(x, y, h_{k}^{-}\right)=V\left(x, y, h_{k}^{+}\right), \\
W\left(x, y, h_{k}^{-}\right)=W\left(x, y, h_{k}^{+}\right), \\
\Phi\left(x, y, h_{k}^{-}\right)=\Phi\left(x, y, h_{k}^{+}\right) .
\end{gathered}
$$

\section{Method of Solution}

Two differential quadrature techniques are employed to reduce the governing equations into a nonlinear eigenvalue problem, as follows.

3.1. Sinc Differential Quadrature Method (SDQM). A cardinal sine function is used as a shape function, such that the unknown $\psi$ and its derivatives can be approximated as a weighted linear sum of nodal values, $\psi_{i},(i=-N, N)$, as follows [27-32]:

$$
\begin{aligned}
\psi\left(x_{i}\right) & =\sum_{j=-N}^{N} \frac{\sin \left[\pi\left(x_{i}-x_{j}\right) / h_{x}\right]}{\pi\left(x_{i}-x_{j}\right) / h_{x}} \psi\left(x_{j}\right), \quad(i=-N, N), h_{x}>0, \\
\left.\frac{\partial \psi}{\partial x}\right|_{x=x_{i}} & =\sum_{j=-N}^{N} A_{i j}^{x} \psi\left(x_{j}\right), \quad(i=-N, N), \\
\left.\frac{\partial^{2} \psi}{\partial x^{2}}\right|_{x=x_{i}} & =\sum_{j=-N}^{N} B_{i j}^{x} \psi\left(x_{j}\right), \quad(i=-N, N),
\end{aligned}
$$

where $\psi$ denotes $U, V, W$, and $\Phi ; N$ is the number of grid points; and $h_{x}$ is the grid size. The weighting coefficients, $A_{i j}^{x}$ and $B_{i j}^{x}$, can be determined by differentiating (28) as follows:

$$
\begin{gathered}
A_{i j}^{x}= \begin{cases}\frac{(-1)^{i-j}}{h_{x}(i-j)}, & i \neq j, \\
0, & i=j,\end{cases} \\
B_{i j}^{x}= \begin{cases}\frac{-2(-1)^{i-j}}{h_{x}^{2}(i-j)^{2}} & i \neq j, \\
\frac{-\pi^{2}}{3 h_{x}^{2}} & i=j .\end{cases}
\end{gathered}
$$

3.2. Discrete Singular Convolution Differential Quadrature Method (DSCDQM). A singular convolution can be defined as follows [33-42]:

$$
F_{(t)}=(T * \eta)(t)=\int_{-\infty}^{\infty} T(t-x) \eta(x) \mathrm{d} x,
$$

where $T(t-x)$ is a singular kernel.

The DSC algorithm can be applied using many types of kernels. These kernels are applied as shape functions, such that the unknown $\psi$ and its derivatives are approximated as a weighted linear sum of $\psi_{i},(i=-N, N)$ over a narrow bandwidth $\left(x-x_{M}, x+x_{M}\right)$ [33-37].

Two kernels of DSC will be employed as follows:

(a) A Delta Lagrange kernel (DLK) can be used as a shape function, such that the unknown $\psi$ and its 
derivatives can be approximated as a weighted linear sum of nodal values, $\psi_{i},(i=-N, N)$, as follows:

$$
\begin{array}{r}
\psi\left(x_{i}\right)=\sum_{j=-M}^{M} \frac{\prod_{k=-M}^{M}\left(x_{i}-x_{k}\right)}{\left(x_{i}-x_{j}\right) \prod_{j=-M, j \neq k}^{M}\left(x_{j}-x_{k}\right)} \psi\left(x_{j}\right), \\
(i=-N, N), M \geq 1,
\end{array}
$$

$$
\begin{aligned}
\left.\frac{\partial \psi}{\partial x}\right|_{x=x_{i}} & =\sum_{j=-M}^{M} A_{i j}^{x} \psi\left(x_{j}\right), \\
\left.\frac{\partial^{2} \psi}{\partial x^{2}}\right|_{x=x_{i}} & =\sum_{j=-M}^{M} B_{i j}^{x} \psi\left(x_{j}\right), \quad(i=-N, N),
\end{aligned}
$$

where $2 M+1$ is the effective computational bandwidth. $A_{i j}^{x}$ and $B_{i j}^{x}$ are defined as follows:

$$
\begin{aligned}
& A_{i j}^{x}= \begin{cases}\frac{1}{\left(x_{i}-x_{j}\right)} \prod_{k=-M, k \neq i, j}^{M} \frac{\left(x_{i}-x_{k}\right)}{\left(x_{j}-x_{k}\right)}, & i \neq j, \\
-\sum_{j=-M, j \neq i}^{-M} A_{i j}^{x}, & i=j,\end{cases} \\
& B_{i j}^{x}= \begin{cases}2\left(A_{i j}^{x} \cdot A_{i i}^{x}-\frac{A_{i j}^{x}}{\left(x_{i}-x_{j}\right)}\right), & i \neq j, \\
-\sum_{j=-M, j \neq i}^{M} B_{i j}^{x}, & i=j .\end{cases}
\end{aligned}
$$

(b) A regularized Shannon kernel (RSK) can also be used as a shape function, such that the unknown $\psi$ and its derivatives can be approximated as a weighted linear sum of nodal values, $\psi_{i},(i=-N, N)$, as follows:

$$
\begin{array}{r}
\psi\left(x_{i}\right)=\sum_{j=-M}^{M}\left\langle\frac{\sin \left[\pi\left(x_{i}-x_{j}\right) / h_{x}\right]}{\pi\left(x_{i}-x_{j}\right) / h_{x}} e^{-\left(\left(x_{i}-x_{j}\right)^{2} / 2 \sigma^{2}\right)}\right\rangle \psi\left(x_{j}\right), \\
(i=-N, N), \sigma=\left(r * h_{x}\right)>0,
\end{array}
$$

$$
\begin{aligned}
& \left.\frac{\partial \psi}{\partial x}\right|_{x=x_{i}}=\sum_{j=-M}^{M} A_{i j}^{x} \psi\left(x_{j}\right), \\
& \left.\frac{\partial^{2} \psi}{\partial x^{2}}\right|_{x=x_{i}}=\sum_{j=-M}^{M} B_{i j}^{x} \psi\left(x_{j}\right), \quad(i=-N, N),
\end{aligned}
$$

where $\sigma$ is a regularization parameter and $r$ is a computational parameter. The weighting coefficients $A_{i j}^{x}$ and $B_{i j}^{x}$ can be defined as follows [33-42]:

$$
\begin{aligned}
& A_{i j}^{x}= \begin{cases}\frac{(-1)^{i-j}}{h_{x}(i-j)} e^{-h_{x}\left((i-j)^{2} / 2 \sigma^{2}\right)}, & i \neq j, \\
0, & i=j,\end{cases} \\
& B_{i j}^{x}= \begin{cases}\left(\frac{2(-1)^{i-j+1}}{h_{x}^{2}(i-j)^{2}}+\frac{1}{\sigma^{2}}\right) e^{-h_{x}^{2}\left((i-j)^{2} / 2 \sigma^{2}\right),} & i \neq j, \\
-\frac{1}{\sigma^{2}}-\frac{\pi^{2}}{3 h_{x}^{2}}, & i=j .\end{cases}
\end{aligned}
$$

Similarly, one can approximate $\psi_{y}, \psi_{z}, \psi_{y y}$, and $\psi_{z z}$ and calculate $A_{i j}^{y}, A_{i j}^{z}, B_{i j}^{y}$, and $B_{i j}^{z}$. Two methods are used to transform the governing equation to linear equations.

3.2.1. Perturbation Method. Assuming the solution of equations (17)-(27) to be a power series that can be solved by a perturbation method of the second order [43] by substituting $W=W_{o}+\varepsilon W_{1}+\varepsilon^{2} W_{2}+\cdots$ into (17)-(27) and equating the terms with the identical powers of $\varepsilon$, where $\mathcal{E}$ is a perturbation parameter:

$$
\begin{aligned}
& C_{11} U_{, x x}+C_{66} U_{, y y}+C_{55} U_{, z z}+\left(C_{12}+C_{66}\right) V_{, x y}+\left(C_{13}+C_{55}\right)\left(W_{o, x z}+\varepsilon W_{1, x z}+\varepsilon^{2} W_{2, x z}\right)+\left(e_{1}+e_{5}\right) \Phi_{, x z}=-\rho \omega^{2} U \\
& \left(C_{66}+C_{12}\right) U_{, x y}+C_{66} V_{, x x}+C_{11} V_{, y y}+C_{44} V_{, z z}+\left(C_{13}+C_{44}\right)\left(W_{o, y z}+\varepsilon W_{1, y z}+\varepsilon^{2} W_{2, y z}\right)+\left(e_{2}+e_{4}\right) \Phi_{, y z}=-\rho \omega^{2} V \\
& \left(C_{55}+C_{13}\right) U_{, x z}+\left(C_{13}+C_{44}\right) V_{, y z}+\left(C_{55}-k_{2}\right)\left(W_{o, x x}+\varepsilon W_{1, x x}+\varepsilon^{2} W_{2, x x}\right)+\left(C_{44}-k_{2}\right)\left(W_{o, y y}+\varepsilon W_{1, y y}+\varepsilon^{2} W_{2, y y}\right) \\
& \quad+C_{33}\left(W_{o, z z}+\varepsilon W_{1, z z}+\varepsilon^{2} W_{2, z z}\right)+e_{5} \Phi_{, x x}+e_{4} \Phi_{, y y}+e_{3} \Phi_{, z z}+k_{1}\left(W_{o}+\varepsilon W_{1}+\varepsilon^{2} W_{2}\right)+k_{3} \varepsilon\left(W_{o}+\varepsilon W_{1}+\varepsilon^{2} W_{2}\right)^{3} \\
& \quad=-\rho \omega^{2}\left(W_{o}+\varepsilon W_{1}+\varepsilon^{2} W_{2}\right) \\
& \left(e_{1}+e_{5}\right) U_{, x z}+\left(e_{2}+e_{4}\right) V_{, y z}+e_{5}\left(W_{o, x x}+\varepsilon W_{1, x x}+\varepsilon^{2} W_{2, x x}\right)+e_{4}\left(W_{o, y y}+\varepsilon W_{1, y y}+\varepsilon^{2} W_{2, y y}\right)+e_{3}\left(W_{o, z z}+\varepsilon W_{1, z z}+\varepsilon^{2} W_{2, z z}\right) \\
& -\eta_{1} \Phi_{, x x}-\eta_{2} \Phi_{, y y}-\eta_{3} \Phi_{, z z}=0 .
\end{aligned}
$$


Equate the terms of $\varepsilon^{0}$ in (39)-(42):

$$
\begin{gathered}
C_{11} U_{, x x}+C_{66} U_{, y y}+C_{55} U_{, z z}+\left(C_{12}+C_{66}\right) V_{, x y}+\left(C_{13}+C_{55}\right) W_{0, x z}+\left(e_{1}+e_{5}\right) \Phi_{, x z}=-\rho \omega^{2} U, \\
\left(C_{66}+C_{12}\right) U_{, x y}+C_{66} V_{, x x}+C_{11} V_{, y y}+C_{44} V_{, z z}+\left(C_{13}+C_{44}\right) W_{0, y z}+\left(e_{2}+e_{4}\right) \Phi_{, y z}=-\rho \omega^{2} V, \\
\left(C_{55}+C_{13}\right) U_{, x z}+\left(C_{13}+C_{44}\right) V_{, y z}+\left(C_{55}-k_{2}\right) W_{o, x x}+\left(C_{44}-k_{2}\right) W_{o, y y}+C_{33} W_{o, z z}+e_{5} \Phi_{, x x}+e_{4} \Phi_{, y y} \\
+e_{3} \Phi_{, z z}+k_{1} W_{o}=-\rho \omega^{2} W_{o}, \\
\left(e_{1}+e_{5}\right) U_{, x z}+\left(e_{2}+e_{4}\right) V_{, y z}+e_{5} W_{0, x x}+e_{4} W_{0, y y}+e_{3} W_{0, z z}-\eta_{1} \Phi_{, x x}-\eta_{2} \Phi_{, y y}-\eta_{3} \Phi_{, z z}=0 .
\end{gathered}
$$

The previous system is an eigenvalue problem, which is solved to obtain the natural frequencies and the bending deflection $W_{0}$.

$$
\begin{gathered}
C_{11} U_{, x x}+C_{66} U_{, y y}+C_{55} U_{, z z}+\left(C_{12}+C_{66}\right) V_{, x y}+\left(C_{13}+C_{55}\right) W_{1, x z}+\left(e_{1}+e_{5}\right) \Phi_{, x z}=-\rho \omega^{2} U \\
\left(C_{66}+C_{12}\right) U_{, x y}+C_{66} V_{, x x}+C_{11} V_{, y y}+C_{44} V_{, z z}+\left(C_{13}+C_{44}\right) W_{1, y z}+\left(e_{2}+e_{4}\right) \Phi_{, y z}=-\rho \omega^{2} V \\
\left(C_{55}+C_{13}\right) U_{, x z}+\left(C_{13}+C_{44}\right) V_{, y z}+\left(C_{55}-k_{2}\right) W_{1, x x}+\left(C_{44}-k_{2}\right) W_{1, y y}+C_{33} W_{1, z z} \\
\quad+e_{5} \Phi_{, x x}+e_{4} \Phi_{, y y}+e_{3} \Phi_{, z z}+\left(k_{1}+\rho \omega^{2}\right) W_{1}=-k_{3} W_{o}^{3}, \\
\left(e_{1}+e_{5}\right) U_{, x z}+\left(e_{2}+e_{4}\right) V_{, y z}+e_{5} W_{1, x x}+e_{4} W_{1, y y}+e_{3} W_{1, z z}-\eta_{1} \Phi_{, x x}-\eta_{2} \Phi_{, y y}-\eta_{3} \Phi_{, z z}=0
\end{gathered}
$$

The previous system is a bending problem, which is solved to obtain $W_{1}$.

Equate the terms of $\varepsilon^{2}$ in (39)-(42):

$$
\begin{gathered}
C_{11} U_{, x x}+C_{66} U_{, y y}+C_{55} U_{, z z}+\left(C_{12}+C_{66}\right) V_{, x y}+\left(C_{13}+C_{55}\right) W_{2, x z}+\left(e_{1}+e_{5}\right) \Phi_{, x z}=-\rho \omega^{2} U, \\
\left(C_{66}+C_{12}\right) U_{, x y}+C_{66} V_{, x x}+C_{11} V_{, y y}+C_{44} V_{, z z}+\left(C_{13}+C_{44}\right) W_{2, y z}+\left(e_{2}+e_{4}\right) \Phi_{, y z}=-\rho \omega^{2} V, \\
\left(C_{55}+C_{13}\right) U_{, x z}+\left(C_{13}+C_{44}\right) V_{, y z}+\left(C_{55}-k_{2}\right) W_{2, x x}+\left(C_{44}-k_{2}\right) W_{2, y y}+C_{33} W_{2, z z}+e_{5} \Phi_{, x x}+e_{4} \Phi_{, y y} \\
+e_{3} \Phi_{, z z}\left(k_{1}+\rho \omega^{2}\right) W_{2}=-3 k_{3} W_{o}^{2} W_{1}, \\
\left(e_{1}+e_{5}\right) U_{, x z}+\left(e_{2}+e_{4}\right) V_{, y z}+e_{5} W_{2, x x}+e_{4} W_{2, y y}+e_{3} W_{2, z z}-\eta_{1} \Phi_{, x x}-\eta_{2} \Phi_{, y y}-\eta_{3} \Phi_{, z z}=0 .
\end{gathered}
$$

The previous system is a bending problem, which is solved to obtain $W_{2}$.

Finally, the series solution can be written as follows:

$$
W_{\text {numerical }}=\lim _{\varepsilon \longrightarrow 1} W=W_{o}+W_{1}+W_{2} .
$$

The convergence condition [43] due to the perturbation method is set as follows:

$$
\left|\frac{W_{i+1}}{W_{i}}\right|<1, \quad \text { where } i=0,1 .
$$

3.2.2. Iterative Quadrature. We solved the following iterative system: 


$$
\begin{aligned}
& C_{11} U_{, x x}+C_{66} U_{, y y}+C_{55} U_{, z z}+\left(C_{12}+C_{66}\right) V_{, x y}+\left(C_{13}+C_{55}\right) W_{i+1, x z}+\left(e_{1}+e_{5}\right) \Phi_{, x z}=-\rho \omega^{2} U, \quad i=0,1,2, \\
& \left(C_{66}+C_{12}\right) U_{, x y}+C_{66} V_{, x x}+C_{11} V_{, y y}+C_{44} V_{, z z}+\left(C_{13}+C_{44}\right) W_{i+1, y z}+\left(e_{2}+e_{4}\right) \Phi_{, y z}=-\rho \omega^{2} V, \quad i=0,1,2, \\
& \quad\left(C_{55}+C_{13}\right) U_{, x z}+\left(C_{13}+C_{44}\right) V_{, y z}+\left(C_{55}-k_{2}\right) W_{i+1, x x}+\left(C_{44}-k_{2}\right) W_{i+1, y y}+C_{33} W_{i+1, z z}+e_{5} \Phi_{, x x} \\
& \quad+e_{4} \Phi_{, y y}+e_{3} \Phi_{, z z}+k_{1} W_{i+1}+k_{3} W_{i}^{2} W_{i+1}=-\rho \omega^{2} W_{i+1}, \quad i=0,1,2, \\
& \left(e_{1}+e_{5}\right) U_{, x z}+\left(e_{2}+e_{4}\right) V_{, y z}+e_{5} W_{i+1, x x}+e_{4} W_{i+1, y y}+e_{3} W_{i+1, z z}-\eta_{1} \Phi_{, x x}-\eta_{2} \Phi_{, y y}-\eta_{3} \Phi_{, z z}=0, \quad i=0,1,2 .
\end{aligned}
$$

To get $W_{o}$, we solved the following eigenvalue problem:

$$
\begin{aligned}
& C_{11} \sum_{l=-N_{x}}^{N_{x}} B_{i l}^{x} U_{l j k}+C_{66} \sum_{m=-N_{y}}^{N_{y}} B_{j m}^{y} U_{i m k}+C_{55} \sum_{n=-N_{z}}^{N_{z}} B_{k n}^{z} U_{i j n}+\left(C_{12}+C_{66}\right) \sum_{l=-N_{x}}^{N_{x}} A_{i l}^{x} \sum_{m=-N_{y}}^{N_{y}} A_{j m}^{y} V_{l m k} \\
&+\left(C_{13}+C_{55}\right) \sum_{l=-N_{x}}^{N_{x}} A_{i l}^{x} \sum_{n=-N_{z}}^{N_{z}} A_{k n}^{z} W_{0, l j n}+\left(e_{1}+e_{5}\right) \sum_{l=-N_{x}}^{N_{x}} A_{i l}^{x} \sum_{n=-N_{z}}^{N_{z}} A_{k n}^{z} \Phi_{l j n}=-\rho \omega^{2} U, \\
&\left(C_{12}+C_{66}\right) \sum_{l=-N_{x}}^{N_{x}} A_{i l}^{x} \sum_{m=-N_{y}}^{N_{y}} A_{j m}^{y} U_{l m k}+C_{66} \sum_{l=-N_{x}}^{N_{x}} B_{i l}^{x} V_{l j k}+C_{11} \sum_{m=-N_{y}}^{N_{y}} B_{j m}^{y} V_{i m k} \\
&+C_{44} \sum_{n=-N_{z}}^{N_{z}} B_{k n}^{z} V_{i j n}+\left(C_{13}+C_{44}\right) \sum_{m=-N_{y}}^{N_{y}} A_{j m}^{y} \sum_{n=-N_{z}}^{N_{z}} A_{k n}^{z} W_{0, i m n}+\left(e_{2}+e_{4}\right) \sum_{m=-N_{y}}^{N_{y}} A_{j m}^{y} \sum_{n=-N_{z}}^{N_{z}} A_{k n}^{z} \Phi_{i m n}=-\rho \omega^{2} V, \\
&\left(C_{55}+C_{13}\right) \sum_{l=-N_{x}}^{N_{x}} A_{i l}^{x} \sum_{n=-N_{z}}^{N_{z}} A_{k n}^{z} U_{l j n}+\left(C_{13}+C_{44}\right) \sum_{m=-N_{y}}^{N_{y}} A_{j m}^{y} \sum_{n=-N_{z}}^{N_{z}} A_{k n}^{z} V_{i m n}+\left(C_{55}-k_{2}\right) \sum_{l=-N_{x}}^{N_{x}} B_{i l}^{x} W_{0, l j k} \\
&+\left(C_{44}-k_{2}\right) \sum_{m=-N_{y}}^{N_{y}} B_{j m}^{y} W_{0, i m k}+C_{33} \sum_{n=-N_{z}}^{N_{z}} B_{k n}^{z} W_{i j n}+e_{5} \sum_{l=-N_{x}}^{N_{x}} B_{i l}^{x} \Phi_{l j k}+e_{4} \sum_{m=-N_{y}}^{N_{y}} B_{j m}^{y} \Phi_{i m k} \\
&+e_{3} \sum_{n=-N_{z}}^{N_{z}} B_{k n}^{z} \Phi_{i j n}+k_{1} W_{0, i j k}=-\rho \omega^{2} W_{0}, \\
&\left.+e_{1}+e_{5}\right) \sum_{l=-N_{x}}^{N_{x}} A_{i l}^{x} \sum_{n=-N_{z}}^{N_{z}} A_{k n}^{z} U_{l j n}+\left(e_{2}+e_{4}\right) \sum_{m=-N_{y}}^{N_{y}} B_{j m}^{y} \sum_{n=-N_{z}}^{N_{y}} A_{k n}^{z} V_{i m n}+e_{5} \sum_{l=-N_{x}}^{N_{x}} B_{i l}^{x} W_{0, l j k} \\
& \\
&+\sum_{n=-N_{z}}+e_{3} \sum_{n=-N_{z}}^{N_{z}} B_{k n}^{z} W_{0, i j n}-\eta_{1} \sum_{l=-N_{x}}^{N_{x}} B_{i l}^{x} \Phi_{l j k}-\eta_{2} \sum_{m=-N_{y}}^{N_{y}} B_{j m}^{y} \Phi_{i m k}-\eta_{3} \sum_{n=-N_{z}}^{N_{z}} B_{k n}^{z} \Phi_{l j n}=0 .
\end{aligned}
$$

The boundary conditions (21)-(27) can also be approximated using two DQMs as follows:

(1) Simply supported (S):

$$
\begin{aligned}
& W_{i j k}=V_{i j k}=C_{11} \sum_{l=-N_{x}}^{N_{x}} A_{i l}^{x} U_{l j k}+C_{12} \sum_{m=-N_{y}}^{N_{y}} A_{j m}^{y} V_{i m k}+C_{13} \sum_{n=-N_{z}}^{N_{z}} A_{k n}^{z} W_{i j n}+e_{1} \sum_{n=-N_{z}}^{N_{z}} A_{k n}^{z} \Phi_{i j n}=0, \quad \text { at } x=0, a, \\
& W_{i j k}=U_{i j k}=C_{12} \sum_{l=-N_{x}}^{N_{x}} A_{i l}^{x} U_{l j k}+C_{11} \sum_{m=-N_{y}}^{N_{y}} A_{j m}^{y} V_{i m k}+C_{13} \sum_{n=-N_{z}}^{N_{z}} A_{k n}^{z} W_{i j n}+e_{2} \sum_{n=-N_{z}}^{N_{z}} A_{k n}^{z} \Phi_{i j n}=0, \quad \text { at } y=0, b .
\end{aligned}
$$


(2) Clamped (C):

$W_{i j k}=V_{i j k}=U_{i j k}=0, \quad$ at $x=0, a y=0, b$.

$$
\begin{aligned}
& C_{11} \sum_{l=-N_{x}}^{N_{x}} A_{i l}^{x} U_{l j k}+C_{12} \sum_{m=-N_{y}}^{N_{y}} A_{j m}^{y} V_{i m k}+C_{13} \sum_{n=-N_{z}}^{N_{z}} A_{k n}^{z} W_{i j n}+e_{1} \sum_{n=-N_{z}}^{N_{z}} A_{k n}^{z} \Phi_{i j n}=0, \\
& C_{55}\left(\sum_{l=-N_{x}}^{N_{x}} A_{i l}^{x} W_{l j k}+\sum_{n=-N_{z}}^{N_{z}} A_{k n}^{z} U_{i j n}\right)+e_{5} \sum_{l=-N_{x}}^{N_{x}} A_{i l}^{x} \Phi_{l j k}=C_{66}\left(\sum_{l=-N_{x}}^{N_{x}} A_{i l}^{x} V_{l j k}+\sum_{m=-N_{y}}^{N_{y}} A_{j m}^{y} U_{i m k}\right)=0, \quad \text { at } x=0, a, \\
& C_{12} \sum_{l=-N_{x}}^{N_{x}} A_{i l}^{x} U_{l j k}+C_{11} \sum_{m=-N_{y}}^{N_{y}} A_{j m}^{y} V_{i m k}+C_{13} \sum_{n=-N_{z}}^{N_{z}} A_{k n}^{z} W_{i j n}+e_{2} \sum_{n=-N_{z}}^{N_{z}} A_{k n}^{z} \Phi_{i j n}=0, \\
& C_{44}\left(\sum_{m=-N_{y}}^{N_{y}} A_{j m}^{y} W_{i m k}+\sum_{n=-N_{z}}^{N_{z}} A_{k n}^{z} V_{i j n}\right)+e_{4} \sum_{m=-N_{y}}^{N_{y}} A_{j m}^{y} \Phi_{i m k}=C_{66}\left(\sum_{l=-N_{x}}^{N_{x}} A_{i l}^{x} V_{l j k}+\sum_{m=-N_{y}}^{N_{y}} A_{j m}^{y} U_{i m k}\right)=0, \quad \text { at } y=0, b,
\end{aligned}
$$

Mechanical and electrical boundary conditions at the lower and upper surfaces of the composite are as follows:

$$
\begin{aligned}
& C_{13} \sum_{l=-N_{x}}^{N_{x}} A_{i l}^{x} U_{l j k}+C_{13} \sum_{m=-N_{y}}^{N_{y}} A_{j m}^{y} V_{i m k}+C_{33} \sum_{n=-N_{z}}^{N_{z}} A_{k n}^{z} W_{i j n}+e_{3} \sum_{n=-N_{z}}^{N_{z}} A_{k n}^{z} \Phi_{i j n}=C_{55}\left(\sum_{l=-N_{x}}^{N_{x}} A_{i l}^{x} W_{l j k}+\sum_{n=-N_{z}}^{N_{z}} A_{k n}^{z} U_{i j n}\right) \\
& \quad+e_{5} \sum_{l=-N_{x}}^{N_{x}} A_{i l}^{x} \Phi_{l j k}=0 \\
& C_{44}\left(\sum_{m=-N_{y}}^{N_{y}} A_{j m}^{y} W_{i m k}+\sum_{n=-N_{z}}^{N_{z}} A_{k n}^{z} V_{i j n}\right)+e_{4} \sum_{m=-N_{y}}^{N_{y}} A_{j m}^{y} \Phi_{i m k}=e_{1} \sum_{l=-N_{x}}^{N_{x}} A_{i l}^{x} U_{l j k}+e_{2} \sum_{m=-N_{y}}^{N_{y}} A_{j m}^{y} V_{i m k}+e_{3} \sum_{n=-N_{z}}^{N_{z}} A_{k n}^{z} W_{i j n} \\
& \quad-\eta_{3} \sum_{n=-N_{z}}^{N_{z}} A_{k n}^{z} \Phi_{i j n}=0,{ }^{2} z=0, \\
& C_{13} \sum_{l=-N_{x}}^{N_{x}} A_{i l}^{x} U_{l j k}+C_{13} \sum_{m=-N_{y}}^{N_{y}} A_{j m}^{y} V_{i m k}+C_{33} \sum_{n=-N_{z}}^{N_{z}} A_{k n}^{z} W_{i j n}+e_{3} \sum_{n=-N_{z}}^{N_{z}} A_{k n}^{z} \Phi_{i j n}=C_{55}\left(\sum_{l=-N_{x}}^{N_{x}} A_{i l}^{x} W_{l j k}+\sum_{n=-N_{z}}^{N_{z}} A_{k n}^{z} U_{i j n}\right) \\
& \quad+e_{5} \sum_{l=1}^{N_{x}} A_{i l}^{x} \Phi_{l j k}=0 \\
& C_{44}\left(\sum_{m=-N_{y}}^{N_{y}} A_{j m}^{y} W_{i m k}+\sum_{n=-N_{z}}^{N_{z}} A_{k n}^{z} V_{i j n}\right)+\sum_{m=-N_{y}}^{N_{y}} A_{j m}^{y} \Phi_{i m k}=\Phi_{i j k}=0, \quad \text { at } z=h .
\end{aligned}
$$

The continuity conditions between the interfaces of layers can be assumed as follows:

$$
\begin{gathered}
W_{i j k}^{1}=W_{i j k}^{2}, \\
V_{i j k}^{1}=V_{i j k}^{2}, \\
U_{i j k}^{1}=U_{i j k}^{2}, \\
\Phi_{i j k}^{1}=\Phi_{i j k}^{2} .
\end{gathered}
$$

We have solved the generalized eigenvalue problem [65]:

$$
K X=\omega^{2} M X,
$$

where $K$ is the coefficient matrix of the previous system, $M$ is the mass matrix and can be diagonal with 0 diagonal elements, and $\omega$ is the free vibration frequencies squared.

For nontrivial solutions for (70), the following determinant should be 0 :

$$
\left|K-\omega^{2} M\right|=0
$$

Equation (71) gives the natural frequencies for the composite plate. 
TABLE 1: Material property of composite piezoelectric plate $[59,61]$.

\begin{tabular}{|c|c|c|c|c|c|c|c|c|c|c|}
\hline \multirow[b]{2}{*}{ Material property } & \multicolumn{3}{|c|}{ Young's moduli $(\mathrm{GPa})$} & \multicolumn{3}{|c|}{ Shear moduli $(\mathrm{GPa})$} & \multicolumn{3}{|c|}{ Poisson's ratios } & \multirow{2}{*}{$\begin{array}{c}\text { Density }\left(\mathrm{kg} / \mathrm{m}^{3}\right) \\
\rho \\
1600\end{array}$} \\
\hline & $\begin{array}{c}E_{2} \\
7\end{array}$ & $\begin{array}{c}E_{1} \\
25 E_{2}\end{array}$ & $\begin{array}{l}E_{3} \\
E_{2}\end{array}$ & $\begin{array}{c}G_{12} \\
0.5 E_{2}\end{array}$ & $\begin{array}{l}G_{13} \\
G_{12}\end{array}$ & $\begin{array}{c}G_{23} \\
0.2 E_{2}\end{array}$ & $\begin{array}{c}v_{12} \\
0.25\end{array}$ & $\begin{array}{c}v_{13} \\
0.03\end{array}$ & $\begin{array}{l}v_{23} \\
0.4\end{array}$ & \\
\hline Metal (aluminum, $\mathrm{Al}$ ) & & 70 & & - & - & - & & 0.3 & & 2702 \\
\hline \multirow[t]{3}{*}{ Ceramic (alumina, $\mathrm{Al}_{2} \mathrm{O}_{3}$ ) } & & 380 & & - & 一 & 一 & & 0.3 & & 3800 \\
\hline & \multicolumn{10}{|c|}{ Effective elastic (GPa) } \\
\hline & $C_{11}$ & $C_{12}$ & $C_{13}$ & $C_{22}$ & $C_{23}$ & $C_{33}$ & $C_{44}$ & $C_{55}$ & & $C_{66}$ \\
\hline Sensor PZT-4 & 139 & 78 & 74 & 139 & 74 & 115 & 25.6 & 25.6 & & 30.5 \\
\hline Sensor $\mathrm{BaTiO}_{3}$ & 166 & 77 & 78 & 166 & 78 & 162 & 43 & 43 & & 44.5 \\
\hline Actuator $\mathrm{Ba}_{2} \mathrm{NaNb}_{5} \mathrm{O}_{15}$ & 239 & 104 & 5 & 247 & 52 & 135 & 65 & 66 & & 76 \\
\hline Actuator PZT-5A & 121 & 77 & 77 & 121 & 111 & 21 & 21 & 21 & & 23 \\
\hline \multirow[t]{2}{*}{ Material property } & \multicolumn{5}{|c|}{ Piezoelectric constants $\left(\mathrm{C} / \mathrm{m}^{2}\right)$} & \multicolumn{3}{|c|}{$\begin{array}{l}\text { Dielectric constants } \\
\quad(\mathrm{F} / \mathrm{m}) * 10^{-9}\end{array}$} & & Density $\left(\mathrm{kg} / \mathrm{m}^{3}\right)$ \\
\hline & $e_{1}$ & $e_{2}$ & $e_{3}$ & $e_{4}$ & $e_{5}$ & $\eta_{1}$ & $\eta_{2}$ & $\eta_{3}$ & & $\rho$ \\
\hline Sensor PZT-4 & -5.2 & -5.2 & 15.1 & 12.7 & 12.7 & 6.5 & 6.5 & 5.6 & & 7500 \\
\hline Sensor $\mathrm{BaTiO}_{3}$ & -4.4 & -4.4 & 18.6 & 11.6 & 11.6 & 11.2 & 11.2 & 12.6 & & 5700 \\
\hline Actuator $\mathrm{Ba}_{2} \mathrm{NaNb}_{5} \mathrm{O}_{15}$ & -0.4 & -0.3 & 4.3 & 3.4 & 2.8 & 19.6 & 2.01 & 0.28 & & 5300 \\
\hline Actuator PZT-5A & -5.4 & -5.4 & 15.8 & 3.4 & 3.4 & 8.11 & 8.11 & 7.34 & & 2330 \\
\hline
\end{tabular}

\section{Numerical Results}

The present numerical results demonstrate the convergence and efficiency of each one of the proposed schemes for vibration analysis of the piezoelectric composite plate $\mathrm{Al} /$ $\mathrm{Al}_{2} \mathrm{O}_{3}$ resting on a nonlinear elastic foundation. For all results, the boundary conditions (65)-(69) are augmented in the governing equations (39)-(42).

For practical purposes, the field quantities are normalized as follows:

$$
\begin{aligned}
\left(U^{*}, V^{*}, W^{*}\right) & =\frac{(U, V, W)}{h}, \\
\left(\sigma_{x}^{*}, \sigma_{y}^{*}, \sigma_{z}^{*}, \tau_{x y}^{*}, \tau_{x z}^{*}, \tau_{y z}^{*}\right) & =\frac{\left(\sigma_{x}, \sigma_{y}, \sigma_{z}, \tau_{x y}, \tau_{x z}, \tau_{y z}\right)}{E_{2}}, \\
C_{i j}^{*} & =\frac{C_{i j}}{E_{2}} \\
x^{*} & =\frac{x}{a}, \\
y^{*} & =\frac{y}{b}, \\
z^{*} & =\frac{z}{h}, \\
h & =h_{s}+h_{c}+h_{a}, \\
h_{a} & =h_{s} \\
D & =\frac{E_{c} h^{3}}{\left(12\left(1-v^{2}\right)\right)} \\
\overline{K_{1}} & =\frac{k_{1} a^{4}}{D}, \\
\overline{K_{2}} & =\frac{k_{2} a^{2}}{D}, \\
\overline{K_{3}} & =\frac{k_{3} a^{4} h^{2}}{D}, \\
D & \\
& \\
&
\end{aligned}
$$

where $U^{*}, V^{*}$, and $W^{*}$ are the normalized amplitudes of displacements, $\sigma_{x}^{*}, \sigma_{y}^{*}$, and $\sigma_{z}^{*}$ are the normalized amplitudes of stresses, $\tau_{x y}^{*}, \tau_{x z}^{*}$, and $\tau_{y z}^{*}$ are the normalized amplitudes of shear stresses, $h_{a}$ and $h_{s}$ are the thicknesses of the actuator and sensor, and $D, \overline{K_{1}}, \overline{K_{2}}$, and $\overline{K_{3}}$ are the flexural rigidity for the bottom layer of composite and the equivalent dimensionless elastic foundation constants.

The computational characteristics of each scheme are adapted to reach accurate results with error of order $\leq 10^{-8}$. The obtained dimensionless frequencies $\Omega, \bar{\omega}$, and $\beta$ are evaluated as follows: $\Omega=\omega h \sqrt{\rho / E}, \bar{\omega}=\omega b / \pi^{2} \sqrt{\rho h / D}$, and $\beta=\omega a^{2} \sqrt{\rho h / D}$, where $\rho$ and $E$ are the density and Young's modulus, respectively.

For the present results, material parameters for the composite are listed in Table 1.

For the sinc DQ scheme, the problem is solved over a regular grid, ranging from $3 \times 5 \times 5$ to $11 \times 5 \times 5$. Table 2 shows the convergence of the obtained results for the isotropic plate, which is in agreement with the exact results in [68] over grid size $\geq 7 \times 5 \times 5$.

For DSCDQ scheme based on Delta Lagrange kernel, the problem is also solved over a uniform grid ranging from $3 \times 5 \times 5$ to $11 \times 5 \times 5$. The bandwidth $2 M+1$ ranges from 3 to 11 .

Table 3 shows the convergence of the obtained fundamental frequency, which is in agreement with the exact results in [68] over grid size $\geq 7 \times 5 \times 5$ and bandwidth $\geq 5$. Table 4 shows that the obtained results are more accurate than those obtained using approximated theories [60, 69]. This table also shows that the execution time of DSCDQMDLK was less than that of sinc DQM.

For the DSCDQ scheme based on the regularized Shannon kernel (RSK), the problem was also solved over a uniform grid ranging from $3 \times 5 \times 5$ to $9 \times 5 \times 5$. The bandwidth $2 M+1$ ranged from 3 to 11 and the regularization parameter $\sigma=r h_{x}$ ranged from $1.0 h_{x}$ to $2.3 h_{x}$, where $h_{x}=1 / N-1$. Table 5 shows the convergence of the obtained fundamental frequency to the exact results in [68] over grid 
TABle 2: Comparison between the normalized fundamental frequencies $\beta=\omega a^{2} \sqrt{\rho_{m} h / D_{m}}$ using the sinc DQM, grid size $N$, and the previous exact and numerical frequencies for the simply supported square isotropic plate $\left(a / h=5, h_{c} / h_{p}=200, \overline{K_{1}}=1000, \overline{K_{2}}=100\right.$, and $\left.\overline{K_{3}}=0\right)$.

\begin{tabular}{|c|c|c|c|c|c|c|c|}
\hline Results & $\begin{array}{c}\text { Normalized frequencies } \\
\qquad N\end{array}$ & $\beta_{1}$ & $\beta_{2}$ & $\beta_{3}$ & $\beta_{4}$ & $\beta_{5}$ & $\beta_{6}$ \\
\hline \multirow{5}{*}{ Sinc DQM } & 3 & 55.9218 & 103.4553 & 124.1464 & 161.3903 & 177.5293 & 186.4058 \\
\hline & 5 & 55.9721 & 103.5484 & 124.2581 & 161.5355 & 177.6891 & 186.5736 \\
\hline & 7 & 56.0369 & 103.6682 & 124.2982 & 161.5391 & 177.6930 & 186.5777 \\
\hline & 9 & 56.0369 & 103.6683 & 124.2982 & 161.5391 & 177.6930 & 186.5777 \\
\hline & 11 & 56.0369 & 103.6683 & 124.2982 & 161.5391 & 177.6930 & 186.5777 \\
\hline \multicolumn{2}{|c|}{ Analytical [68] } & 56.0359 & - & - & - & - & - \\
\hline \multicolumn{2}{|c|}{ RHSDT [69] } & 56.0310 & - & - & - & - & - \\
\hline \multicolumn{2}{|c|}{ NSDT [60] } & 56.0311 & - & - & - & - & - \\
\hline \multicolumn{2}{|c|}{ Execution time (sec) } & \multicolumn{5}{|c|}{5.615225 over $N=7 \times 5 \times 5$} & \\
\hline
\end{tabular}

RHSDT: refined hyperbolic shear deformation theory; NSDT: new shear deformation theory; DQM: differential quadrature method.

TABLE 3: Comparison between the normalized fundamental frequency using DSCDQM-DLK, bandwidth $(2 M+1)$, and grid size $N$ for the simply supported square isotropic plate $\left(h_{c} / h_{p}=200, a / h=5, \overline{K_{1}}=1000, \overline{K_{2}}=100\right.$, and $\left.\overline{K_{3}}=0\right)$.

\begin{tabular}{|c|c|c|c|c|c|c|}
\hline \multicolumn{4}{|c|}{ Fundamental frequency } & \multicolumn{3}{|c|}{ SCDQM-DLK } \\
\hline Bandwidth & $N$ & 3 & 5 & 7 & 9 & 11 \\
\hline $2 M+1=3$ & & 55.9844 & 56.0849 & 56.0685 & 56.0685 & 56.0685 \\
\hline $2 M+1=5$ & & 55.9524 & 56.0349 & 56.0320 & 56.0320 & 56.0320 \\
\hline $2 M+1=7$ & & 56.1798 & 56.0650 & 56.0320 & 56.0320 & 56.0320 \\
\hline $2 M+1=9$ & & 55.9384 & 56.2877 & 56.0320 & 56.0320 & 56.0320 \\
\hline $2 M+1=11$ & & 55.9384 & 56.2877 & 56.0320 & 56.0320 & 56.0320 \\
\hline
\end{tabular}

TABLE 4: Comparison between the normalized frequencies $\beta=\omega a^{2} \sqrt{\rho_{m} h / D_{m}}$, using DSCDQM-DLK, grid sizes $N$, and the previous exact and numerical frequencies for the simply supported square isotropic plate $\left(2 M+1=5, h_{c} / h_{p}=200, a / h=5, \overline{K_{1}}=1000, \overline{K_{2}}=100\right.$, and $\left.\overline{K_{3}}=0\right)$.

\begin{tabular}{|c|c|c|c|c|c|c|c|}
\hline \multicolumn{2}{|c|}{ Normalized frequencies } & \multirow{2}{*}{$\beta_{1}$} & \multirow{2}{*}{$\beta_{2}$} & \multirow{2}{*}{$\beta_{3}$} & \multirow{2}{*}{$\beta_{4}$} & \multirow{2}{*}{$\beta_{5}$} & \multirow{2}{*}{$\beta_{6}$} \\
\hline Results & $N$ & & & & & & \\
\hline \multirow{4}{*}{ DSCDQM-DLK } & 3 & 55.9524 & 103.5119 & 124.2142 & 161.4660 & 177.6126 & 186.4932 \\
\hline & 5 & 56.0349 & 103.6085 & 124.3302 & 161.5049 & 177.6553 & 186.5380 \\
\hline & 7 & 56.0320 & 103.6031 & 124.3237 & 161.4964 & 177.6444 & 186.5266 \\
\hline & 9 & 56.0320 & 103.6031 & 124.3237 & 161.4964 & 177.6444 & 186.5266 \\
\hline Analytical [68] & & 56.0359 & - & - & - & - & - \\
\hline RHSDT [69] & & 56.0310 & - & - & - & - & - \\
\hline NSDT [60] & & 56.0311 & - & - & - & - & - \\
\hline \multicolumn{2}{|c|}{ Execution time (sec) } & \multicolumn{6}{|c|}{5.611001 over $N=7 \times 5 \times 5$} \\
\hline
\end{tabular}

TABLE 5: Comparison between the normalized fundamental frequency $\beta$ using the discrete singular convolution differential quadrature method on regularized Shannon kernel (DSCDQM-RSK), bandwidth $(2 M+1)$, regularization parameter $\sigma$, and grid size $N$ for the simply supported square isotropic plate $\left(h_{c} / h_{p}=200, a / h=5, \overline{K_{1}}=1000, \overline{K_{2}}=100\right.$, and $\left.\overline{K_{3}}=0\right)$.

\begin{tabular}{|c|c|c|c|c|c|}
\hline \multirow{2}{*}{$\begin{array}{l}\text { Fundamental frequency } \\
N\end{array}$} & \multirow{2}{*}{$\begin{array}{l}\text { Regularization parameter } \\
\qquad 2 M+1\end{array}$} & \multicolumn{4}{|c|}{ DSCDQM-RSK } \\
\hline & & $\sigma=1 \times h_{x}$ & $\sigma=1.5 \times h_{x}$ & $\sigma=1.9 \times h_{x}$ & $\sigma=2.3 \times h_{x}$ \\
\hline \multirow{3}{*}{3} & 3 & 55.9564 & 55.9468 & 55.9853 & 56.0821 \\
\hline & 5 & 55.9561 & 55.8991 & 55.9780 & 56.0706 \\
\hline & 7 & 55.9561 & 55.8991 & 55.9780 & 56.0706 \\
\hline \multirow{3}{*}{5} & 3 & 55.8758 & 55.9715 & 56.0318 & 56.0318 \\
\hline & 5 & 55.8758 & 55.9715 & 56.0318 & 56.0318 \\
\hline & 7 & 55.8758 & 55.9715 & 56.0318 & 56.0318 \\
\hline \multirow{3}{*}{7} & 3 & 55.8507 & 55.9392 & 56.0318 & 56.0318 \\
\hline & 5 & 55.9563 & 55.9790 & 56.0318 & 56.0318 \\
\hline & 7 & 55.9926 & 55.9917 & 56.0318 & 56.0318 \\
\hline \multirow{3}{*}{9} & 3 & 55.9259 & 55.9857 & 56.0318 & 56.0318 \\
\hline & 5 & 55.9823 & 55.9733 & 56.0318 & 56.0318 \\
\hline & 7 & 55.9506 & 56.0193 & 56.0318 & 56.0318 \\
\hline
\end{tabular}


TABLE 6: Comparison between the normalized frequencies $\beta=\omega a^{2} \sqrt{\rho_{m} h / D_{m}}$ using DSCDQM-RSK, grid size, and the previous exact and numerical frequencies for the simply supported square isotropic plate $\left(2 M+1=3, \sigma=1.9^{*} h_{x}, h_{c} / h_{p}=200, a / h=5, \overline{K_{1}}=1000, \overline{K_{2}}=100\right.$, and $\left.\overline{K_{3}}=0\right)$.

\begin{tabular}{|c|c|c|c|c|c|c|c|}
\hline \multicolumn{2}{|c|}{ Normalized frequencies } & \multirow{2}{*}{$\beta_{1}$} & \multirow[b]{2}{*}{$\beta_{2}$} & \multirow[b]{2}{*}{$\beta_{3}$} & \multirow[b]{2}{*}{$\beta_{4}$} & \multirow[b]{2}{*}{$\beta_{5}$} & \multirow[b]{2}{*}{$\beta_{6}$} \\
\hline Results & $N$ & & & & & & \\
\hline \multirow{4}{*}{ DSCDQM-RSK } & 3 & 55.9853 & 103.5168 & 124.2201 & 161.4861 & 177.6347 & 186.5164 \\
\hline & 5 & 56.0318 & 103.6027 & 124.3232 & 161.6201 & 177.7821 & 186.6712 \\
\hline & 7 & 56.0318 & 103.6027 & 124.3232 & 161.6201 & 177.7821 & 186.6712 \\
\hline & 9 & 56.0318 & 103.6027 & 124.3232 & 161.6201 & 177.7821 & 186.6712 \\
\hline \multicolumn{2}{|l|}{ Analytical [68] } & 56.0359 & - & - & - & - & - \\
\hline \multicolumn{2}{|l|}{ RHSDT [69] } & 56.0310 & - & - & - & - & - \\
\hline \multicolumn{2}{|l|}{ NSDT [60] } & 56.0311 & - & - & - & - & - \\
\hline \multicolumn{3}{|c|}{ Execution time (sec) } & \multicolumn{3}{|c|}{1.933552 over $N=5 \times 5 \times 5$} & & \\
\hline
\end{tabular}

TABle 7: Comparison between the normalized fundamental frequency $\beta=\omega a^{2} \sqrt{\rho_{m} h / D_{m}}$ and the elastic foundation parameter for the simply supported squared plate $\left(h_{c} / h_{p}=200\right.$ and $\left.a / h=5\right)$.

\begin{tabular}{|c|c|c|c|c|}
\hline$\overline{\overline{K_{1}}}, \overline{K_{2}}$ & Nonlinear treatment methods & $\overline{K_{3}}=0$ & $\overline{K_{3}}=50$ & $\overline{K_{3}}=100$ \\
\hline \multirow{5}{*}{0,0} & Perturbation & 17.4590 & 17.4590 & 17.4590 \\
\hline & Execution time (sec) & 0.480763 & 1.2346 & 1.2210 \\
\hline & Iterative quadrature & 17.4590 & 17.4590 & 17.4590 \\
\hline & Execution time (sec) & 0.480763 & 0.743408 & 0.818601 \\
\hline & Analytical [68] & 17.5055 & - & - \\
\hline \multirow{5}{*}{100,10} & Perturbation & 24.2807 & 24.2807 & 24.2807 \\
\hline & Execution time (sec) & 0.469989 & 1.1744 & 1.2081 \\
\hline & Iterative quadrature & 24.2807 & 24.2807 & 24.2807 \\
\hline & Execution time (sec) & 0.469989 & 0.629148 & 0.651756 \\
\hline & Analytical [68] & 24.3074 & - & - \\
\hline \multirow{5}{*}{1000,100} & Perturbation & 56.0318 & 56.0318 & 56.0318 \\
\hline & Execution time (sec) & 0.439478 & 1.1496 & 1.1351 \\
\hline & Iterative quadrature & 56.0318 & 56.0318 & 56.0318 \\
\hline & Execution time (sec) & 0.439478 & 0.620471 & 0.572076 \\
\hline & Analytical [68] & 56.0359 & - & - \\
\hline
\end{tabular}

TABLE 8: Comparison between the normalized fundamental frequency $\beta$, elastic foundation parameter, and different boundary conditions for the square plate $\left(h_{c} / h_{p}=200, a / h=5\right.$, and $\left.\overline{K_{3}}=50\right)$.

\begin{tabular}{|c|c|c|c|c|c|c|}
\hline$\overline{\overline{K_{1}}}$ & $\overline{\overline{K_{2}}}$ & Nonlinear treatment methods & SSSS & SCSC & SSSC & SSSF \\
\hline \multirow{8}{*}{0} & \multirow[b]{2}{*}{0} & Perturbation & 19.7349 & 28.9157 & 23.6398 & 11.6883 \\
\hline & & Iterative quadrature & 19.7349 & 28.9157 & 23.6398 & 11.6883 \\
\hline & \multirow{3}{*}{100} & Perturbation & 48.6329 & 54.6505 & 51.3365 & 37.1656 \\
\hline & & Iterative quadrature & 48.6329 & 54.6505 & 51.3365 & 37.1656 \\
\hline & & Perturbation & 141.9431 & 146.7559 & 144.2712 & 111.7952 \\
\hline & \multirow{3}{*}{1000} & Execution time (sec) & 1.24152 & 1.2158 & 1.22158 & 1.187421 \\
\hline & & Iterative quadrature & 141.9425 & 146.7553 & 144.2706 & 111.7947 \\
\hline & & Execution time (sec) & 0.784509 & 0.70355 & 0.76487 & 0.69457 \\
\hline \multirow{8}{*}{100} & \multirow{2}{*}{0} & Perturbation & 22.1402 & 30.6741 & 25.6818 & 15.3870 \\
\hline & & Iterative quadrature & 22.1402 & 30.6741 & 25.6818 & 15.3870 \\
\hline & \multirow{3}{*}{100} & Perturbation & 49.6324 & 55.5689 & 52.2925 & 38.4718 \\
\hline & & Iterative quadrature & 49.6324 & 55.5689 & 52.2925 & 38.4718 \\
\hline & & Perturbation & 142.2904 & 147.1665 & 144.7015 & 112.3010 \\
\hline & \multirow{3}{*}{1000} & Execution time (sec) & 1.24115 & 1.1187 & 1.24005 & 1.12571 \\
\hline & & Iterative quadrature & 142.3024 & 147.1790 & 144.7098 & 112.3075 \\
\hline & & Execution time (sec) & 0.77785 & 0.70254 & 0.75714 & 0.70478 \\
\hline \multirow{8}{*}{1000} & \multirow[b]{2}{*}{0} & Perturbation & 37.2791 & 42.8293 & 39.4889 & 33.7090 \\
\hline & & Iterative quadrature & 37.2791 & 42.8293 & 39.4889 & 33.7090 \\
\hline & \multirow{3}{*}{100} & Perturbation & 57.9569 & 63.1485 & 60.8547 & 48.6837 \\
\hline & & Iterative quadrature & 57.9569 & 63.1485 & 60.8547 & 48.6837 \\
\hline & & Perturbation & 145.3461 & 150.1200 & 147.6716 & 116.1315 \\
\hline & \multirow{3}{*}{1000} & Execution time (sec) & 1.14287 & 1.11276 & 1.01451 & 0.99475 \\
\hline & & Iterative quadrature & 145.4915 & 150.2702 & 147.6863 & 116.2471 \\
\hline & & Execution time (sec) & 0.75415 & 0.70125 & 0.74583 & 0.69573 \\
\hline
\end{tabular}


TABLE 9: Comparison between the normalized frequencies $\beta=\omega a^{2} \sqrt{\rho_{c} h / D_{c}}$, thickness ratio $(a / h)$, elastic foundation parameter, and different boundary conditions for the square plate $\left(h_{c} / h_{p}=200, \overline{K_{2}}=10\right.$, and $\left.\overline{K_{3}}=50\right)$.

\begin{tabular}{|c|c|c|c|c|c|c|c|}
\hline \multirow{2}{*}{$a / h$} & \multirow{2}{*}{$\overline{K_{1}}$} & \multicolumn{3}{|c|}{ SCSC } & \multicolumn{3}{|c|}{ SCSS } \\
\hline & & $\overline{\omega_{1}}$ & $\overline{\omega_{2}}$ & $\overline{\omega_{3}}$ & $\overline{\omega_{1}}$ & $\overline{\omega_{2}}$ & $\overline{\omega_{3}}$ \\
\hline \multirow{4}{*}{$10 / 3$} & 0 & 2.3150 & 3.4276 & 3.6742 & 2.1762 & 2.4572 & 3.5834 \\
\hline & 10 & 2.3328 & 3.4280 & 3.6324 & 2.1905 & 2.4574 & 3.5909 \\
\hline & 100 & 2.4834 & 3.4311 & 3.6325 & 2.3415 & 2.4616 & 3.7124 \\
\hline & 1000 & 3.4298 & 3.4392 & 3.6326 & 3.3221 & 3.1212 & 3.8330 \\
\hline \multirow{4}{*}{2} & 0 & 1.7629 & 2.2383 & 2.7214 & 1.7072 & 1.8167 & 2.6100 \\
\hline & 10 & 1.7752 & 2.2883 & 2.7623 & 1.7132 & 1.8232 & 2.6112 \\
\hline & 100 & 1.8589 & 2.2885 & 2.7628 & 1.7921 & 1.8672 & 2.6194 \\
\hline & 1000 & 2.0977 & 2.3384 & 2.7456 & 2.0492 & 1.9688 & 2.6414 \\
\hline \multirow{4}{*}{5} & 0 & 2.3897 & 4.7139 & 7.7506 & 2.2456 & 3.3940 & 7.5180 \\
\hline & 10 & 2.4116 & 4.7240 & 7.7562 & 2.3392 & 3.9209 & 7.5247 \\
\hline & 100 & 2.6001 & 4.8135 & 7.8054 & 2.4180 & 3.9989 & 7.5918 \\
\hline & 1000 & 3.9708 & 5.5944 & 8.2594 & 2.4181 & 4.0015 & 7.5921 \\
\hline
\end{tabular}

TABLE 10: Comparison between the normalized frequencies and the thickness $h$ for the clamped plate $\left(a / h=4, \overline{k_{1}}=1000, \overline{k_{2}}=100\right.$, and $\left.\overline{k_{3}}=50\right)$.

\begin{tabular}{|c|c|c|c|c|c|c|}
\hline $\begin{array}{l}\text { Normalized frequencies } \\
H\end{array}$ & $\beta_{1}$ & $\beta_{2}$ & $\beta_{3}$ & $\beta_{4}$ & $\beta_{5}$ & $\beta_{6}$ \\
\hline 0.05 & 79.648 & 164.334 & 211.562 & 237.039 & 301.984 & 327.423 \\
\hline 0.06 & 79.918 & 164.853 & 211.828 & 237.510 & 302.594 & 327.996 \\
\hline 0.07 & 79.918 & 164.854 & 211.853 & 237.512 & 302.594 & 328.010 \\
\hline 0.075 & 79.918 & 164.854 & 211.853 & 237.512 & 302.594 & 328.010 \\
\hline 0.08 & 79.918 & 164.854 & 211.853 & 237.512 & 302.594 & 328.010 \\
\hline 0.09 & 79.918 & 164.854 & 211.853 & 237.512 & 302.594 & 328.010 \\
\hline 0.1 & 79.918 & 164.854 & 211.853 & 237.512 & 302.594 & 328.010 \\
\hline
\end{tabular}

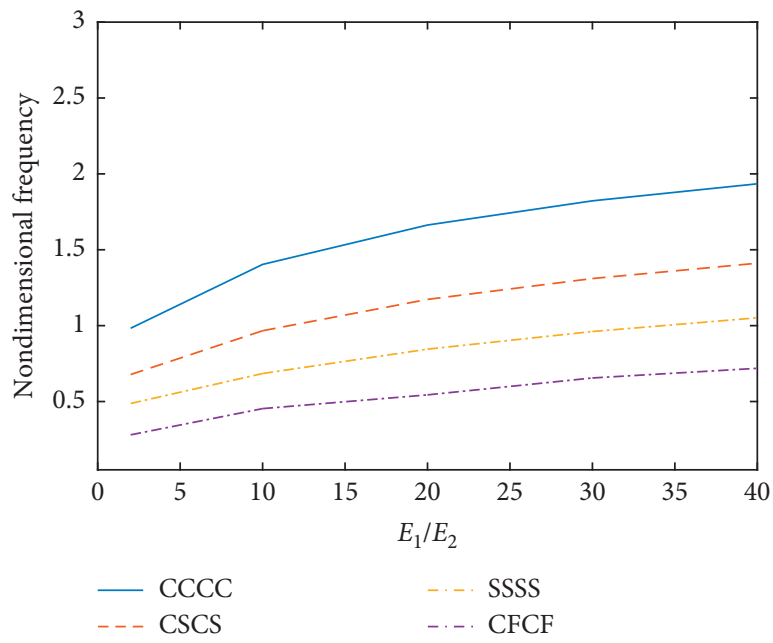

(a)

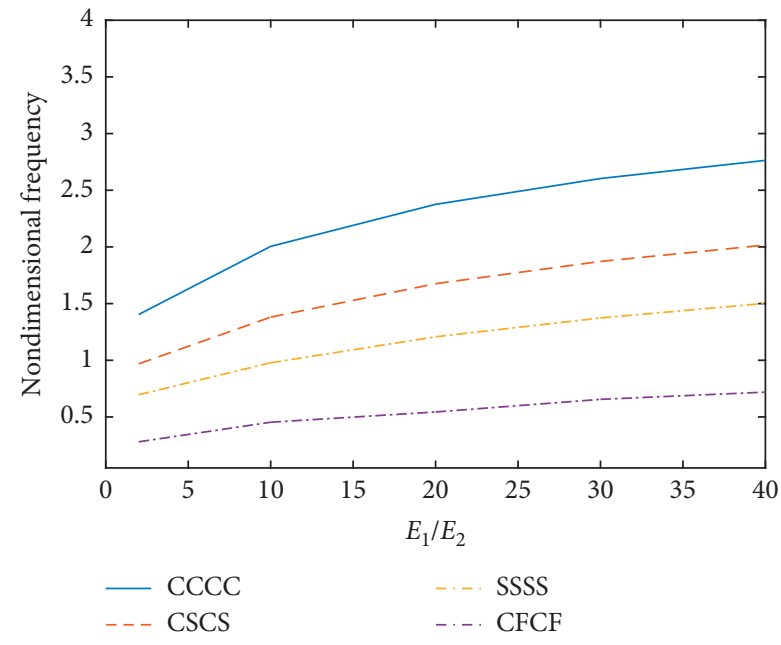

(b)

FIGURE 2: Variation of the fundamental frequency $(\Omega)$ with Young's modulus $\left(E_{1} / E_{2}\right)$, different boundary conditions, and different materials for the square plate $\left(\overline{K_{1}}=1000, \overline{K_{2}}=100, \overline{K_{3}}=50, h_{c} / h_{p}=45\right.$, and $\left.a / h=10\right)$. (a) Sensor is PZT- 4 and actuator is $\mathrm{Ba}_{2} \mathrm{NaNb}_{5} \mathrm{O}_{15} ;(\mathrm{b})$ sensor is $\mathrm{BaTiO}_{3}$ and actuator is PZT-5A.

size $\geq 5 \times 5 \times 5$, bandwidth $\geq 3$, and regularization parameter $\sigma=1.9 h_{x}$.

Table 6 shows that the obtained results from DQ schemes were more accurate than other models in the literature $[60,69]$. Further, the execution time of this scheme is the least. Therefore, the DSCDQM-RSK scheme is the best choice among the examined quadrature schemes for vibration analysis of piezoelectric composite materials resting on nonlinear elastic foundations. Furthermore, a parametric study is introduced to investigate the influence of foundation 


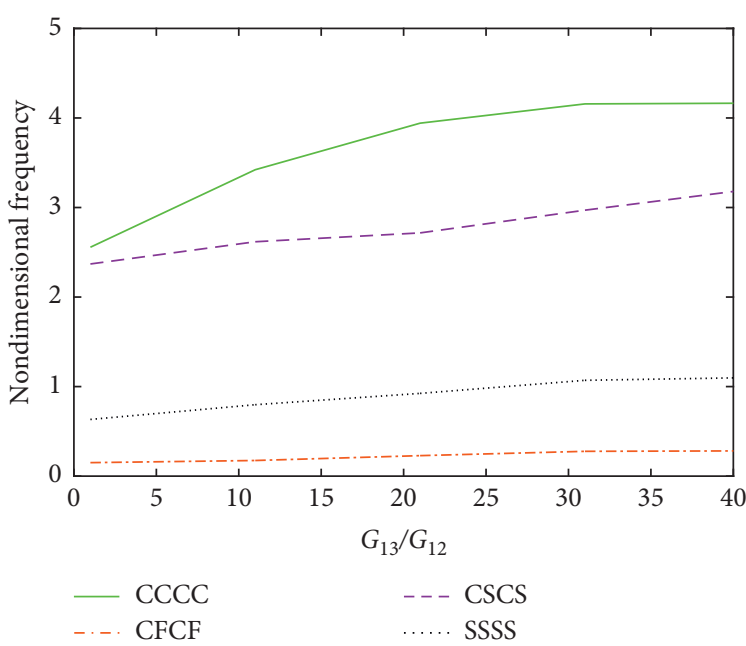

(a)

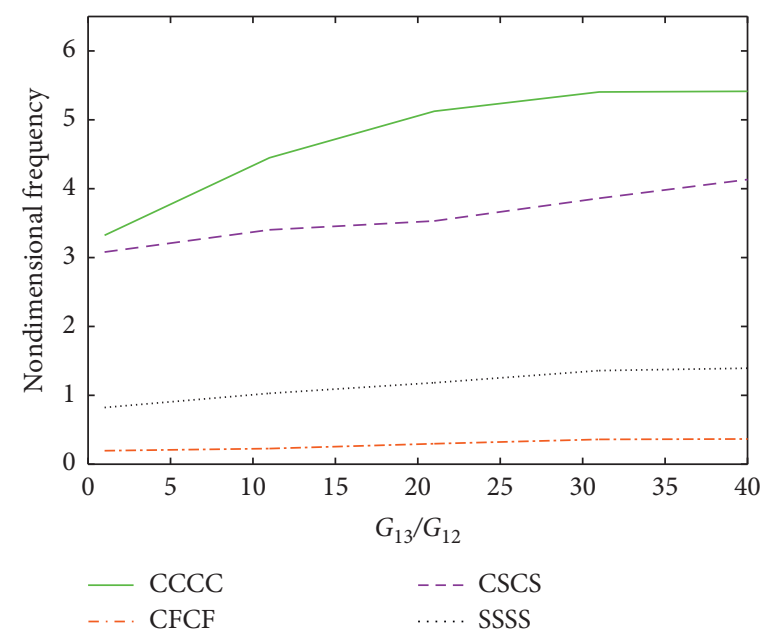

(b)

Figure 3: Variation of the fundamental frequency $(\Omega)$ with shear modulus $\left(G_{13} / G_{12}\right)$, different boundary conditions, and different materials of the square plate $\left(\overline{K_{1}}=1000, \overline{K_{2}}=100, \overline{K_{3}}=50, h_{c} / h_{p}=45\right.$, and $\left.a / h=10\right)$. (a) Sensor is PZT- 4 and actuator is $\mathrm{Ba}_{2} \mathrm{NaNb}_{5} \mathrm{O}_{15} ;(\mathrm{b})$ sensor is $\mathrm{BaTiO}_{3}$ and actuator is PZT-5A.

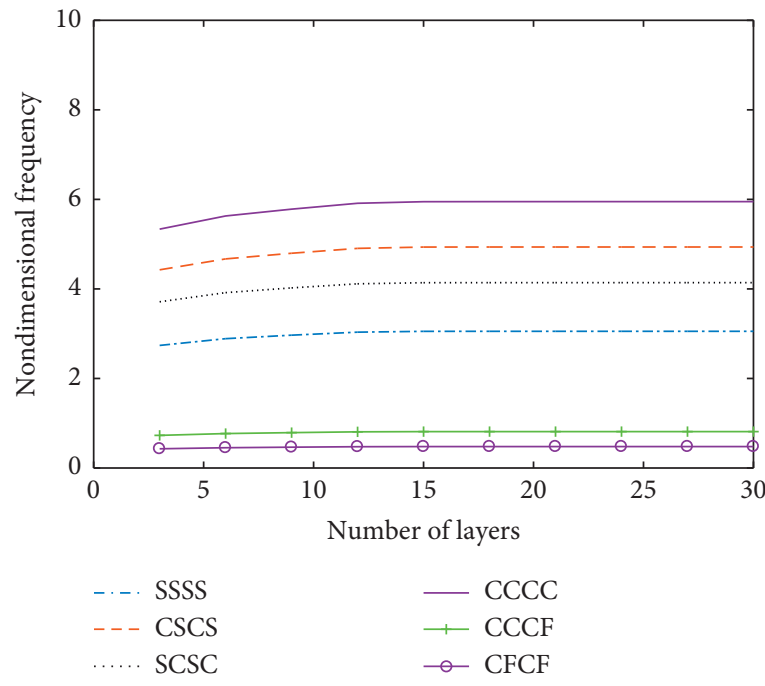

(a)

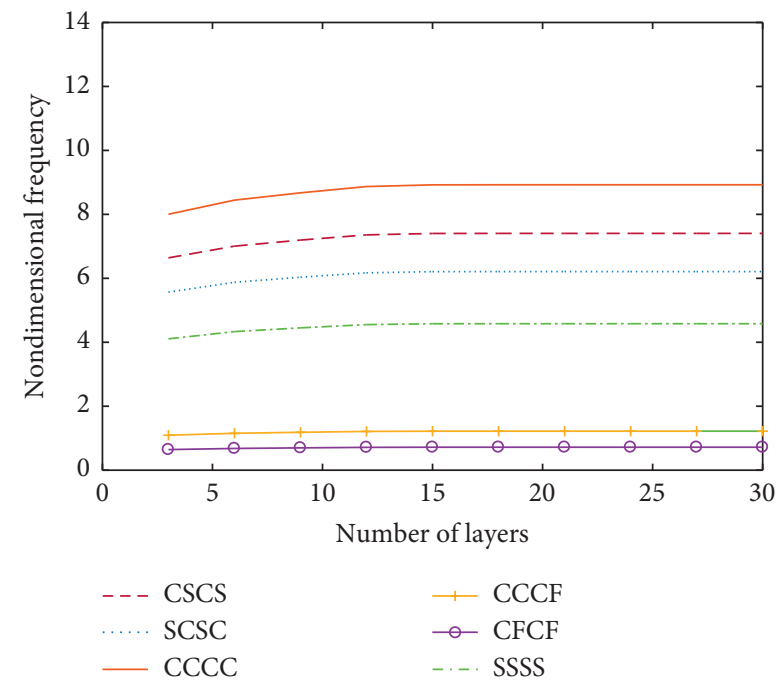

(b)

Figure 4: Variation of the fundamental frequency $(\Omega)$ with the number of layers, different boundary conditions, and different materials of the square plate $\left(\overline{K_{1}}=1000, \overline{K_{2}}=100, \overline{K_{3}}=100, h_{c} / h_{p}=45\right.$, and $\left.a / h=5\right)$. (a) Sensor is PZT- 4 and actuator is $\mathrm{Ba}_{2} \mathrm{NaNb}_{5} \mathrm{O}_{15} ;(\mathrm{b})$ sensor is $\mathrm{BaTiO}_{3}$ and actuator is PZT-5A.

parameters, elastic and geometric characteristics of the composite, and types of material on the values of natural frequencies and mode shapes. Tables 7 and 8 show the natural frequency for isotropic plates. These tables show the effect of elastic foundation parameters on the frequency for different boundary conditions. The natural frequency increased with the increase in foundation parameters $\overline{K_{1}}$ and $\overline{K_{2}}$. Additionally, the SCSC plate had the highest frequency and the SSSF had the lowest frequency. Moreover, the efficiency of the nonlinear treatment methods was investigated. The execution time for the iterative quadrature method was less than the perturbation method, with a factor close to 1.5 until 1.9 for all boundary conditions used in Table 8.

Tables $8-10$ and Figures $2-5$ show that the natural frequencies increase with increasing side-to-thickness ratio $(a / h)$, foundation parameters $\overline{K_{1}}, \overline{K_{2}}, \overline{K_{3}}$, total thickness, Young's modulus gradation ratio $\left(E_{1} / E_{2}\right)$, shear modulus gradation ratio $\left(G_{13} / G_{12}\right)$, and number of layers. The natural frequencies converge at a total thickness $(h=0.075)$ in the 


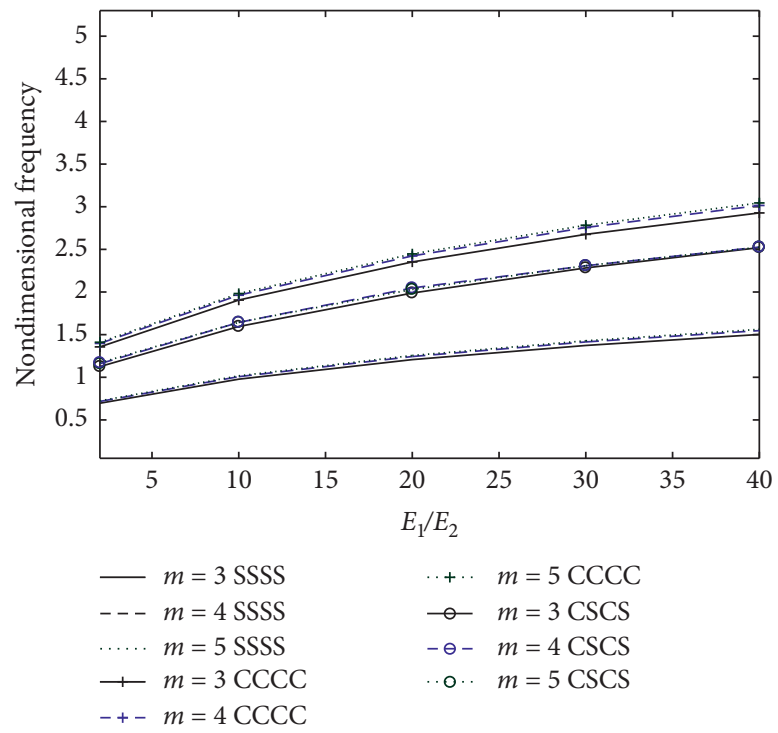

(a)

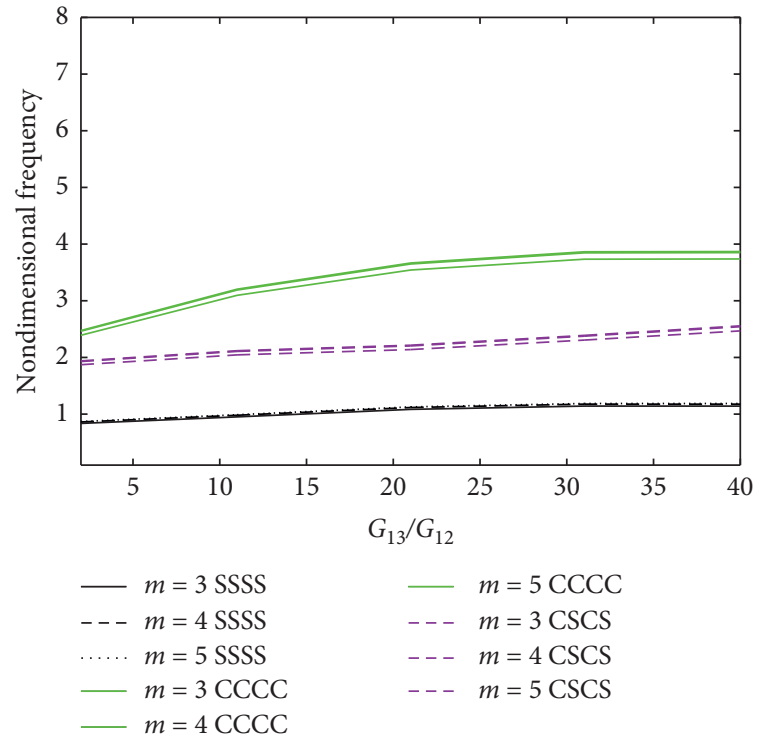

(b)

Figure 5: Variation of the fundamental frequency $(\Omega)$ with Young's modulus $\left(E_{1} / E_{2}\right)$, shear modulus $\left(G_{13} / G_{12}\right)$, and different number of layers for the different materials (sensor is $\mathrm{BaTiO}_{3}$ and actuator is PZT-5A) of the square plate $\left(\overline{K_{1}}=1000, \overline{K_{2}}=100, \overline{K_{3}}=100\right.$, $h_{c} / h_{p}=45$, and $\left.a / h=10\right)$.

TABLE 11: Comparison between the normalized frequencies and thickness $h$ for the simply supported plate $\left(a / h=4, \overline{k_{1}}=1000, \overline{k_{2}}=100\right.$, and $\left.\overline{k_{3}}=50\right)$.

\begin{tabular}{lccccc}
\hline $\begin{array}{l}\text { Normalized frequencies } \\
H\end{array}$ & $\beta_{1}$ & $\beta_{2}$ & $\beta_{3}$ & $\beta_{4}$ & $\beta_{5}$ \\
\hline 0.01 & 51.6122 & 92.90196 & 113.5468 & 144.5142 & 165.159 \\
0.02 & 51.7874 & 93.21732 & 113.9323 & 145.0047 & 165.7197 \\
0.03 & 51.7875 & 93.2175 & 113.9325 & 145.005 & 165.7200 \\
0.035 & 51.7875 & 93.2175 & 113.9325 & 145.005 & 165.7200 \\
0.04 & 51.7875 & 93.2175 & 113.9325 & 145.005 & 185.6427 \\
0.05 & 51.7875 & 93.2175 & 113.9325 & 145.005 & 185.7200 \\
0.07 & 51.7875 & 93.2175 & 113.9325 & 145.005 & 165.7200 \\
\hline
\end{tabular}

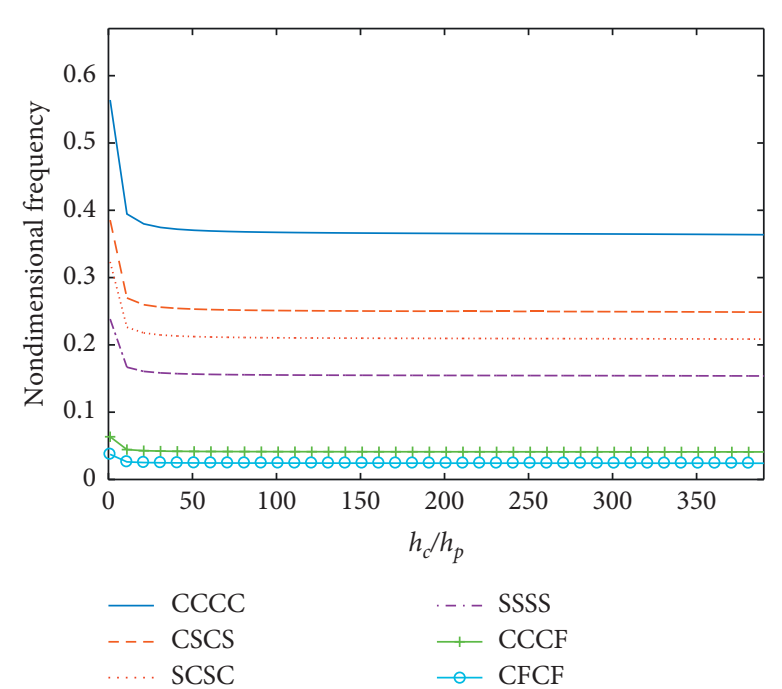

(a)

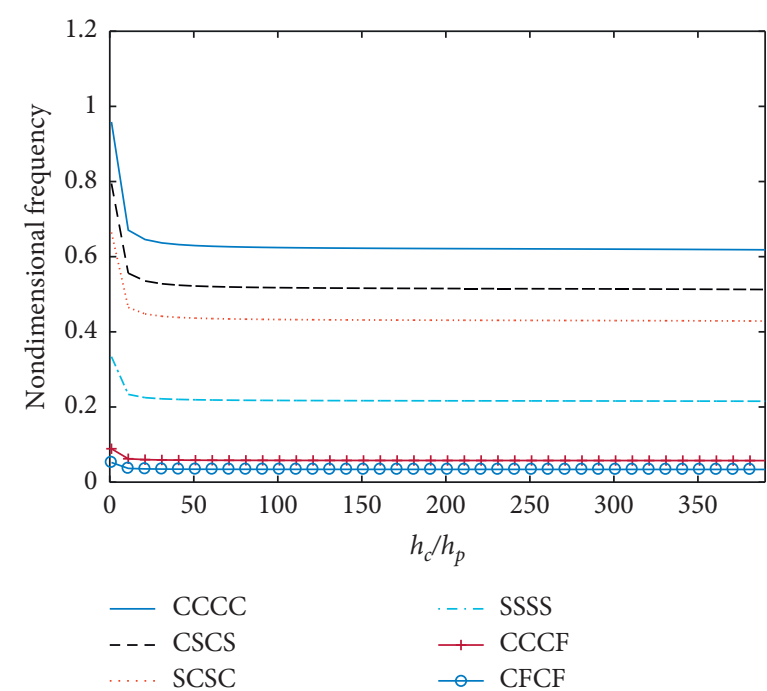

(b)

FiguRE 6: Variation of the fundamental frequency $(\Omega)$ with the composite layer thickness to piezoelectric thickness ratio $\left(h_{\mathrm{c}} / h_{\mathrm{p}}\right)$, length-tothickness ratio $(a / h)$, and different boundary conditions for the square plate $\left(\overline{K_{1}}=1000, \overline{K_{2}}=100, \overline{K_{3}}=50\right.$, and $\left.E_{1} / E_{2}=25\right)$. (a) $a / h=5$ and (b) $a / h=10$. 


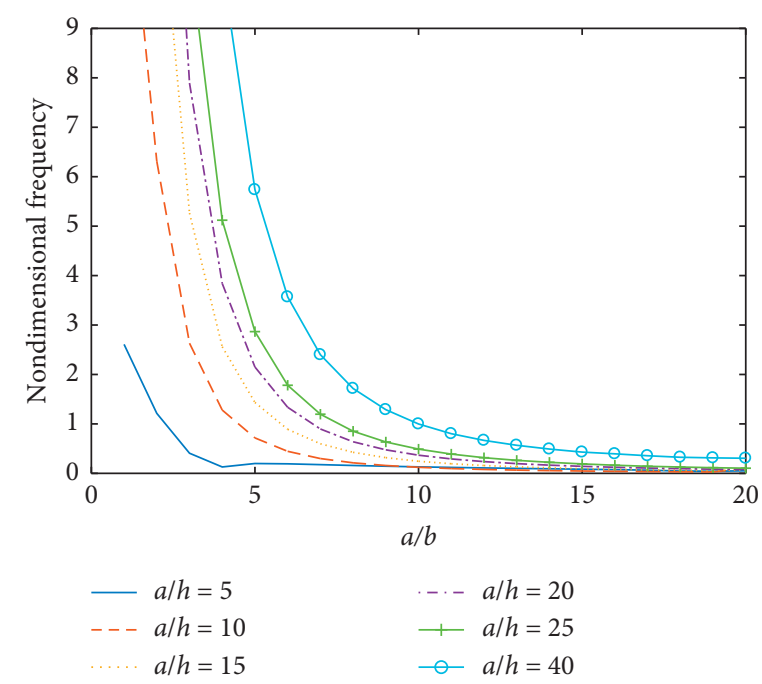

(a)

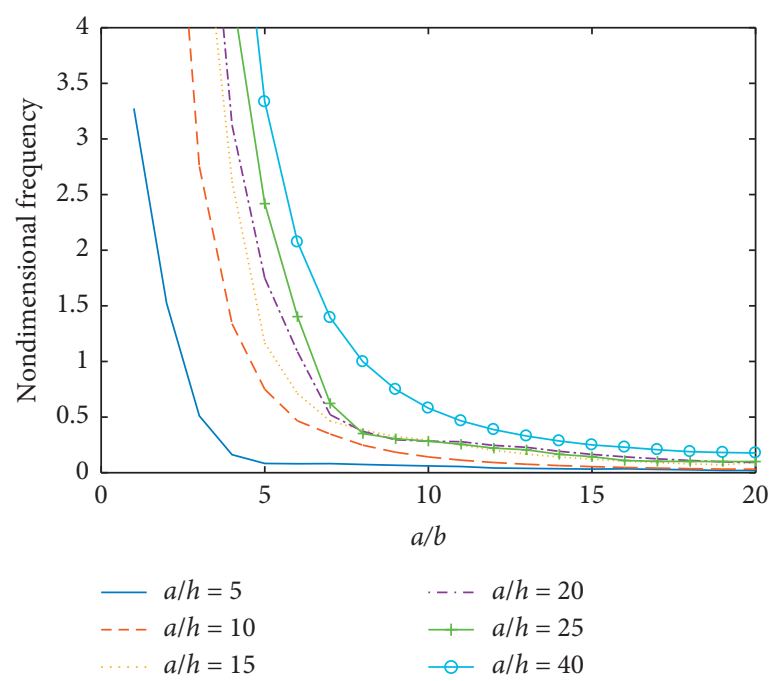

(c)

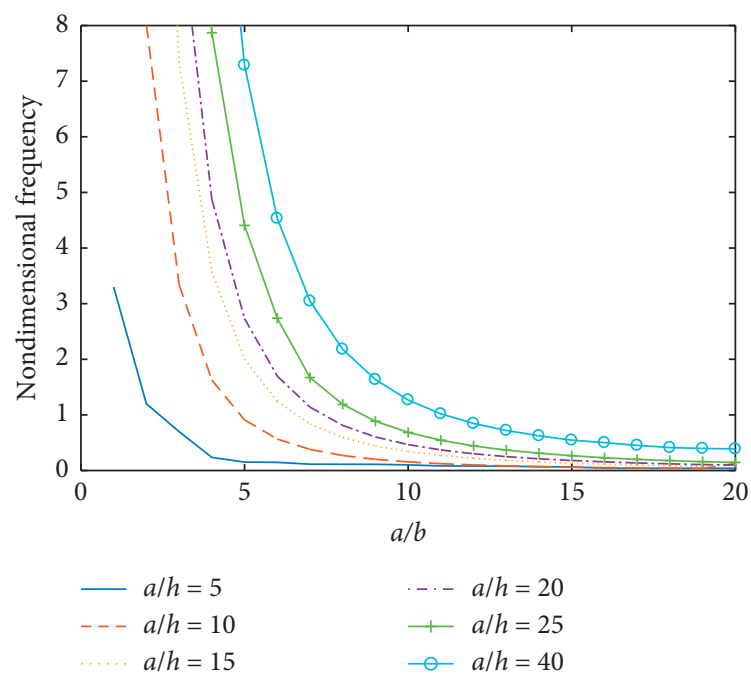

(b)

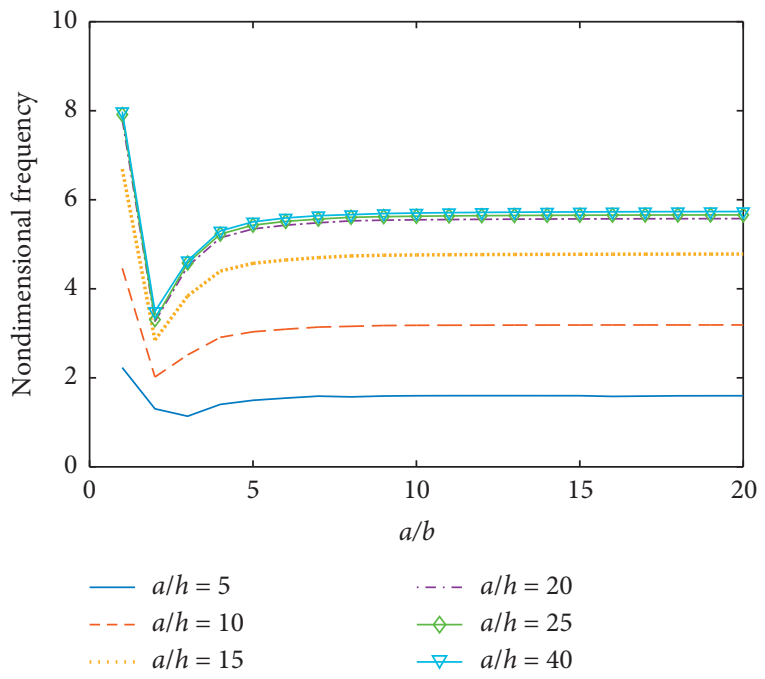

(d)

FIGURE 7: Variation of the fundamental frequency $(\Omega)$ with the aspect ratio $(a / b)$, different values of $a / h$, and different boundary conditions for the square plate $\left(\overline{K_{1}}=1000, \overline{K_{2}}=100, \overline{K_{3}}=100, h_{c} / h_{p}=25\right.$, and $\left.E_{1} / E_{2}=25\right)$. (a) SSSS, (b) CSCS, (c) SCSC, and (d) CCCF.

CCCC plate and a total thickness $(h=0.035)$ in the SSSSS plate, as shown in Tables 10 and 11. Figure 6 shows the natural frequencies decrease with the decrease of the piezoelectric layer thickness $\left(h_{c} / h_{p}\right)$.

Furthermore, Figure 7 shows that the natural frequencies decreased with an increase in the aspect ratio $(a / b)$ at different values $(a / h)$. Moreover, the natural frequencies remained constant when $h_{c} / h_{p}>20$ in all different edge conditions. Figures 2, 3, and 8 show the effect of the material type on the natural frequency. It can be seen that the sensor was more significant than the actuators. The natural frequency was almost unchanged when $a / h>40$. Additionally, the influence of the $\mathrm{BaTiO}_{3}$ material was more affected by the natural frequencies than the PZT-4 material. Furthermore, Figures 9-12 show the first three mode shapes of normalized transverse displacement $(w)$ and electric potential $(\varphi)$. From the previous figures, it can be seen that the normalized transverse displacement was at a maximum for the CCCF plate and was at a minimum for the CCCC plate. Additionally, the electric potential was at a maximum for the CCCF plate and at a minimum for the CSCS plate. Furthermore, the previous figures show that the amplitude of displacement and electric potential increased with the increase of the nonlinear Winkler parameter $\overline{K_{3}}$. 


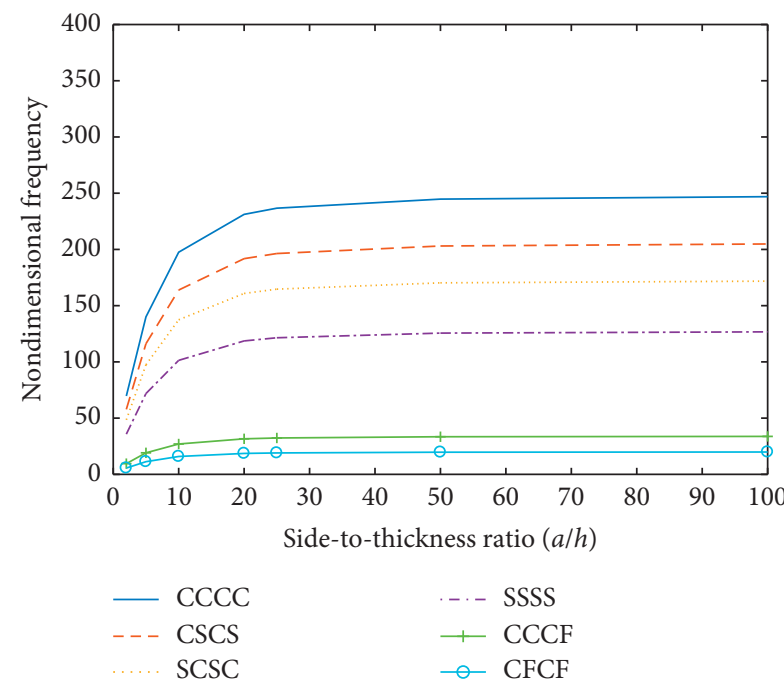

(a)

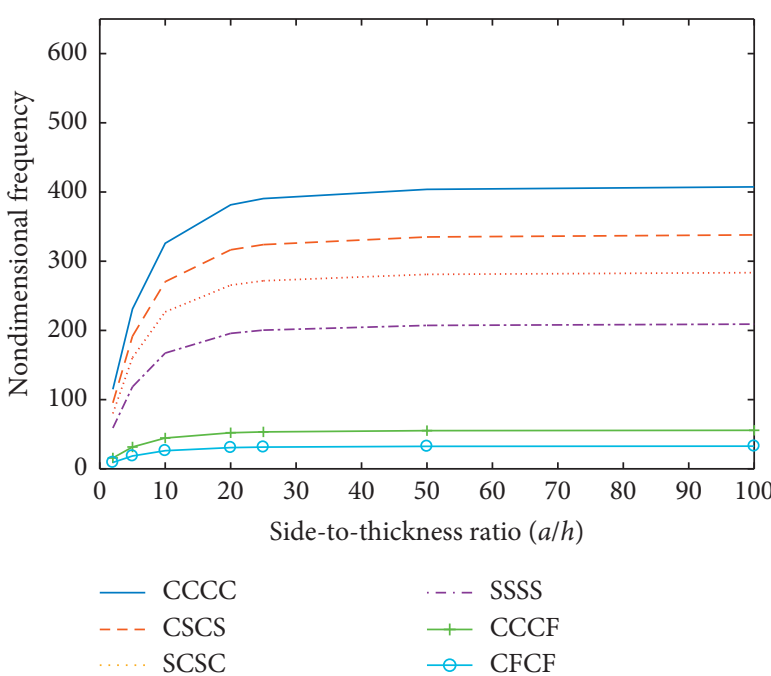

(b)

FiguRE 8: Variation of the fundamental frequency $(\Omega)$ with the side-to-thickness ratio $(a / h)$, different boundary conditions, and different materials for the square plate $\left(\overline{K_{1}}=1000, \overline{K_{2}}=100, \overline{K_{3}}=100\right.$, and $\left.E_{1} / E_{2}=25\right)$. (a) Sensor is $\mathrm{BaTiO}_{3}$ and actuator is $\mathrm{Ba}_{2} \mathrm{NaNb}_{5} \mathrm{O}_{15} ;($ b) sensor is $\mathrm{BaTiO}_{3}$ and actuator is PZT-5A.

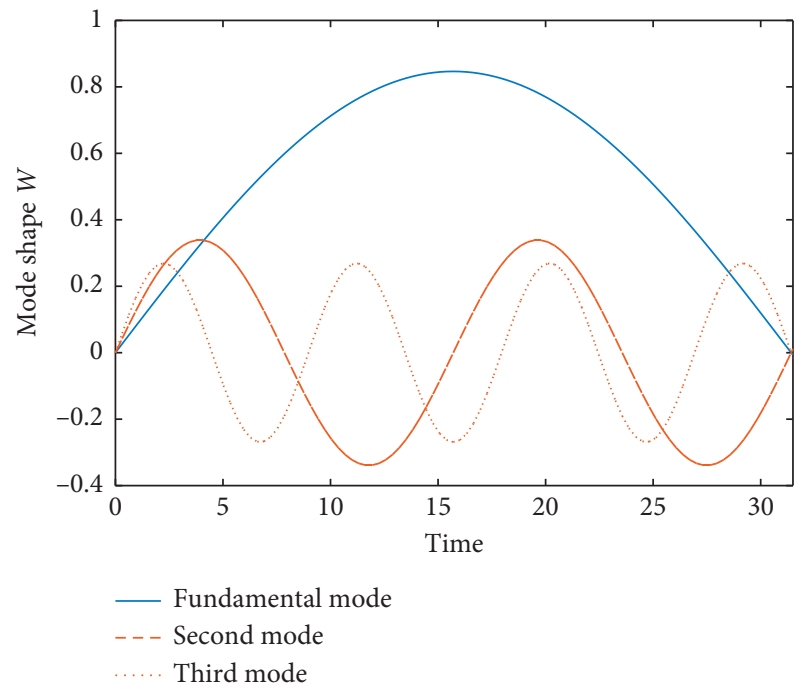

(a)

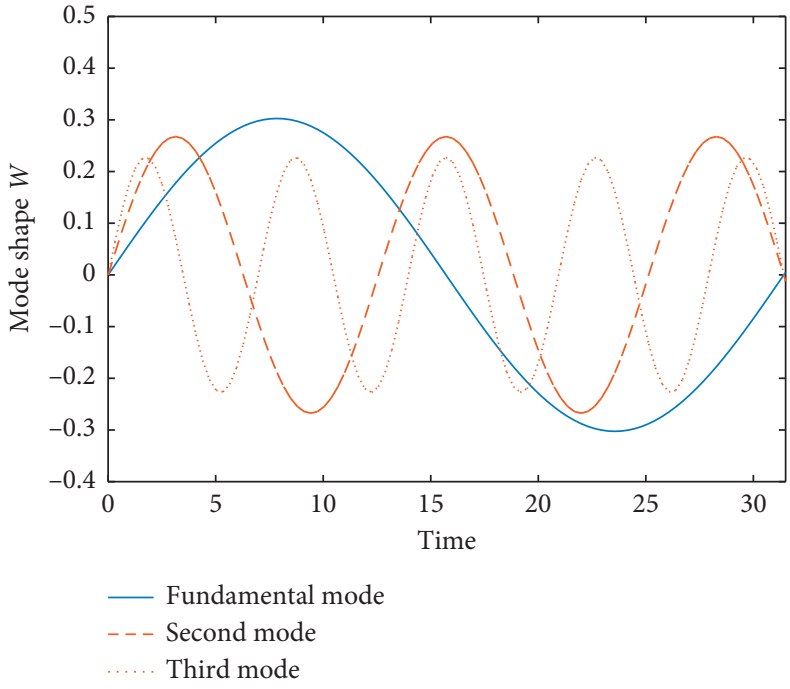

(b)

FIgURE 9: Continued. 


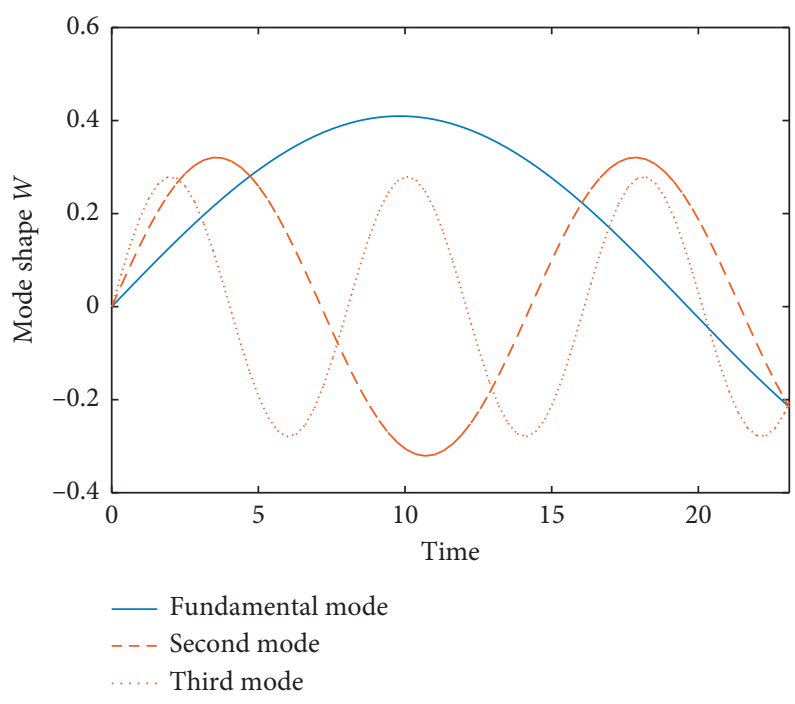

(c)

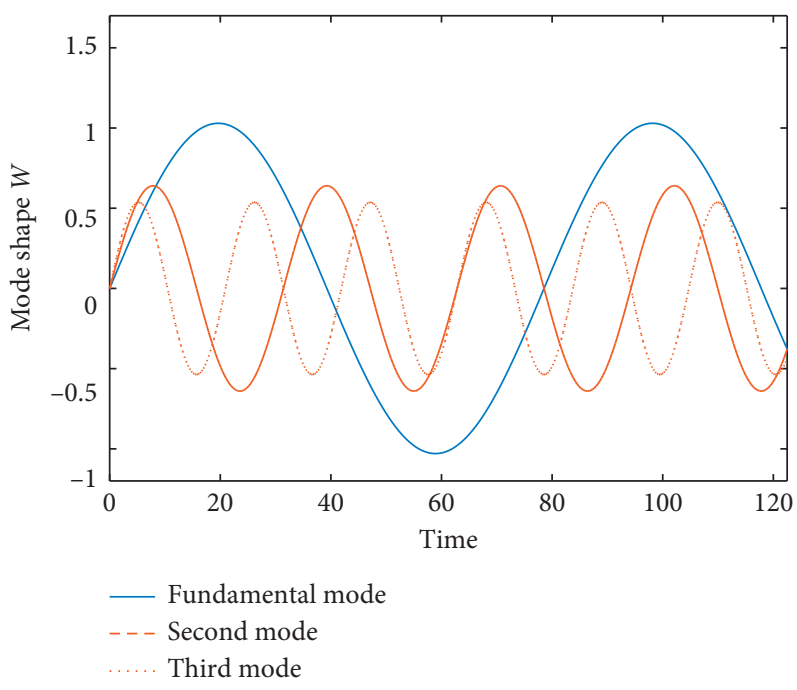

(d)

FIGURE 9: Variation of normalized mode shape deflection $W$ with the time for the first three modes at different boundary conditions for the square plate $\left(\overline{K_{1}}=1000, \overline{K_{2}}=100, \overline{K_{3}}=0, h_{c} / h_{p}=25, E_{1} / E_{2}=25\right.$, and $\left.a / h=10\right)$. (a) SSSS, (b) CCCC, (c) SCSC, and (d) CCCF.

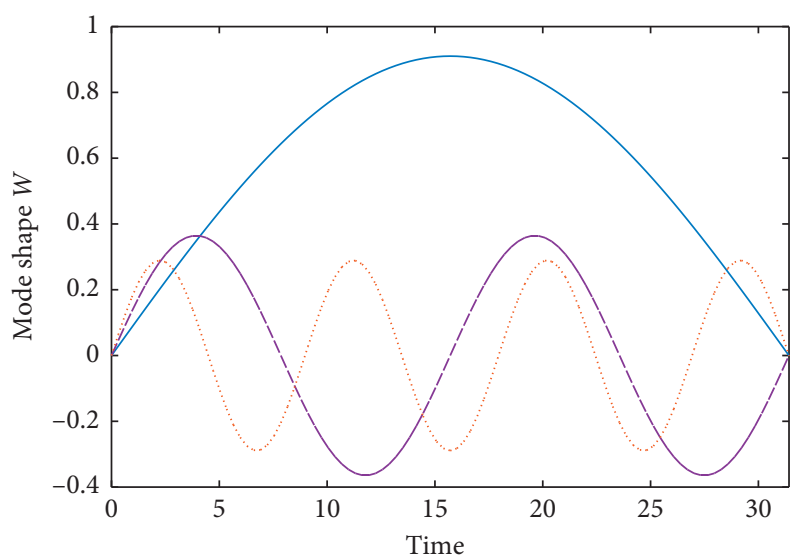

Fundamental mode

Second mode

Third mode

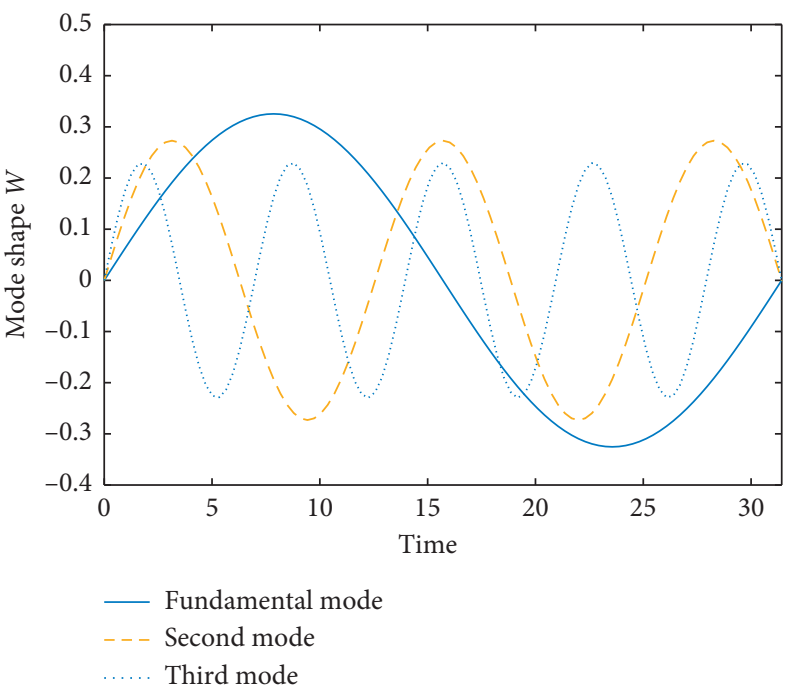

(b)

Figure 10: Continued. 


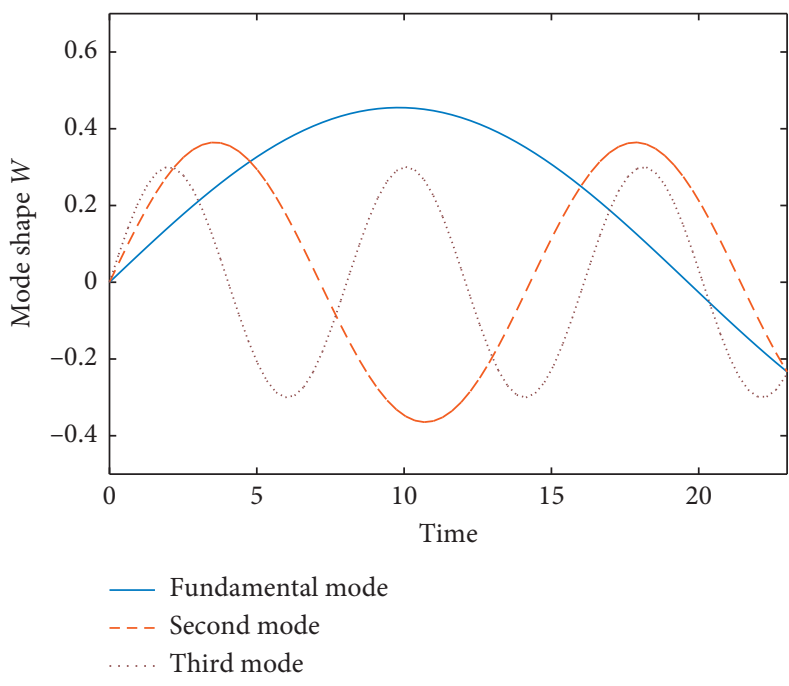

(c)

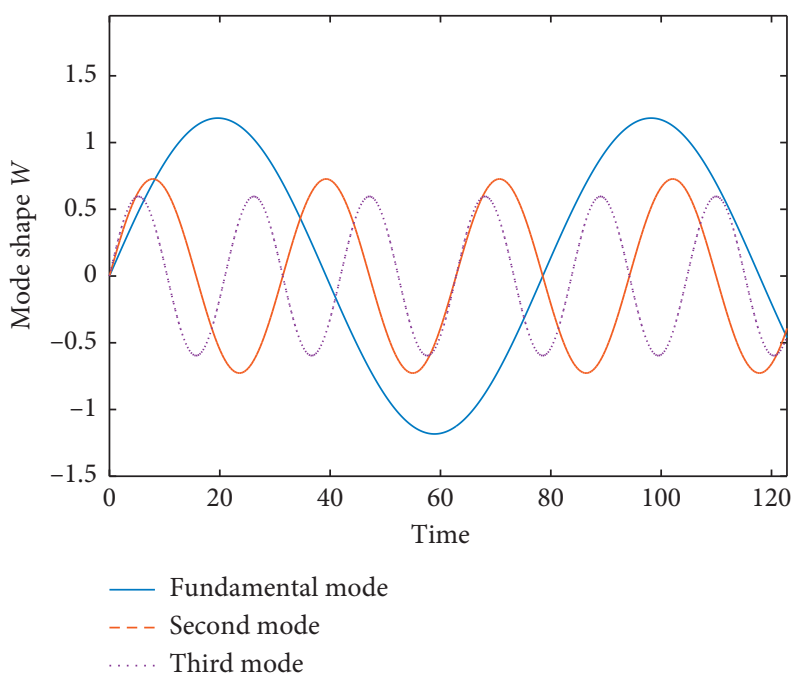

(d)

FIGURE 10: Variation of normalized mode shape deflection $W$ with the time for the first three modes at different boundary conditions for the square plate $\left(\overline{K_{1}}=1000, \overline{K_{2}}=100, \overline{K_{3}}=100, h_{c} / h_{p}=25, E_{1} / E_{2}=25\right.$, and $\left.a / h=10\right)$. (a) SSSS, (b) CCCC, (c) SCSC, and (d) CCCF.

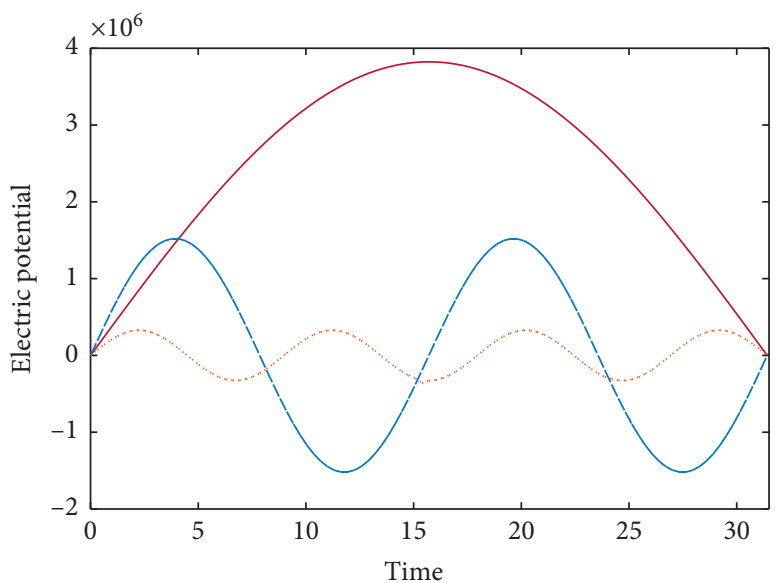

Fundamental mode Second mode Third mode

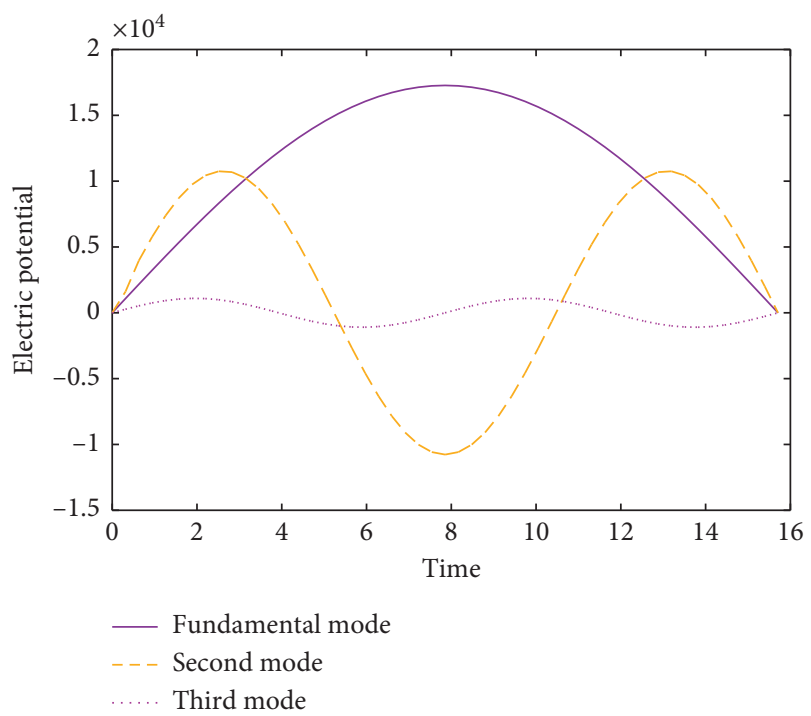

(b)

Figure 11: Continued. 


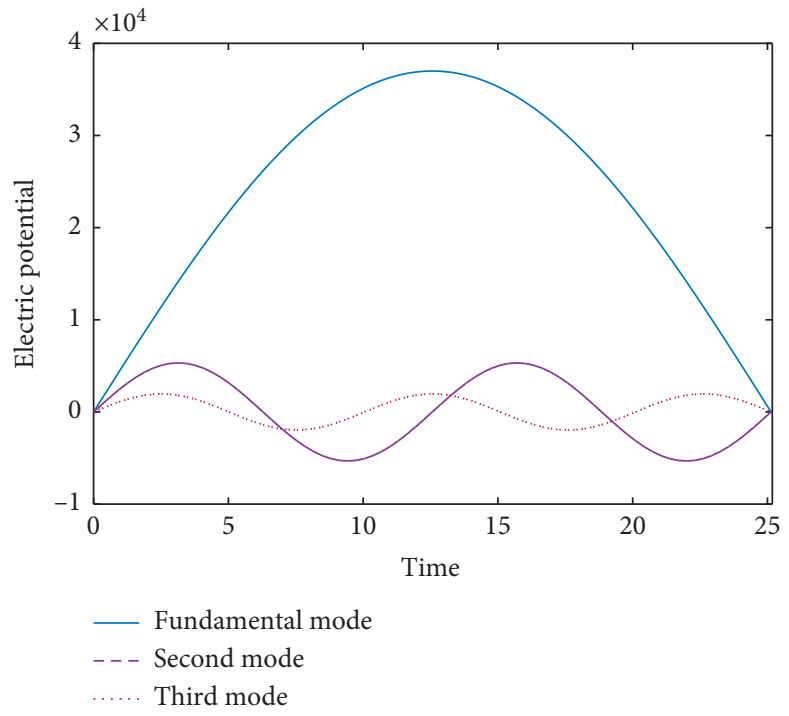

(c)

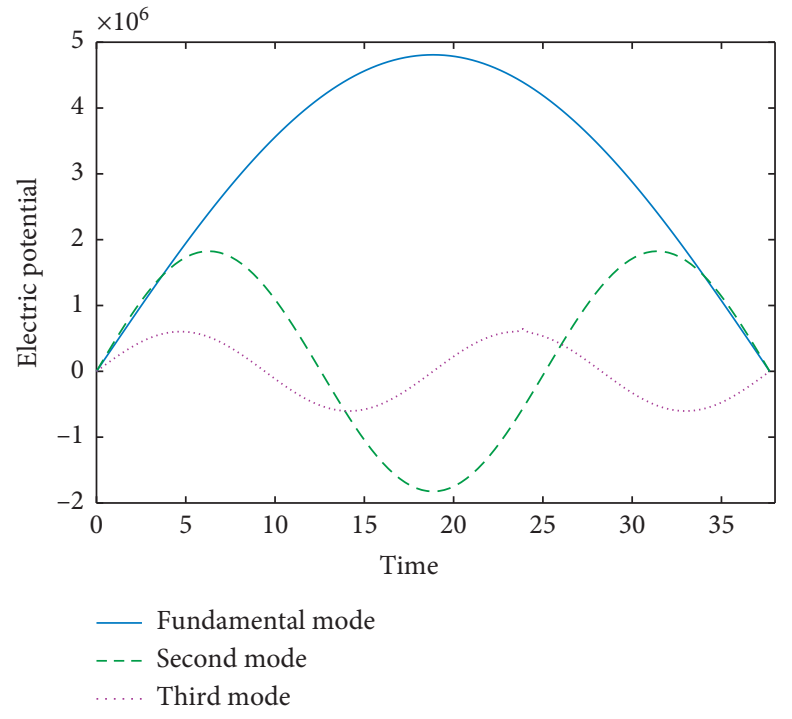

(d)

FIGURE 11: Variation of electric potential with the time for the first three modes at different boundary conditions for the square plate $\left(\overline{K_{1}}=1000, \overline{K_{2}}=100, \overline{K_{3}}=0, h_{c} / h_{p}=25, E_{1} / E_{2}=25\right.$, and $\left.a / h=10\right)$. (a) SSSS, (b) CCCC, (c) SCSC, and (d) CCCF.

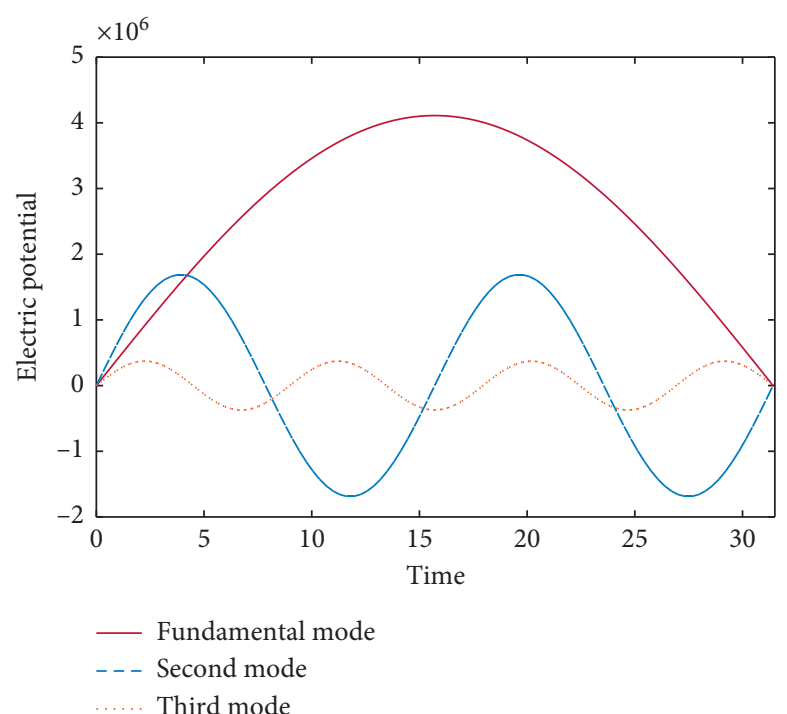

(a)

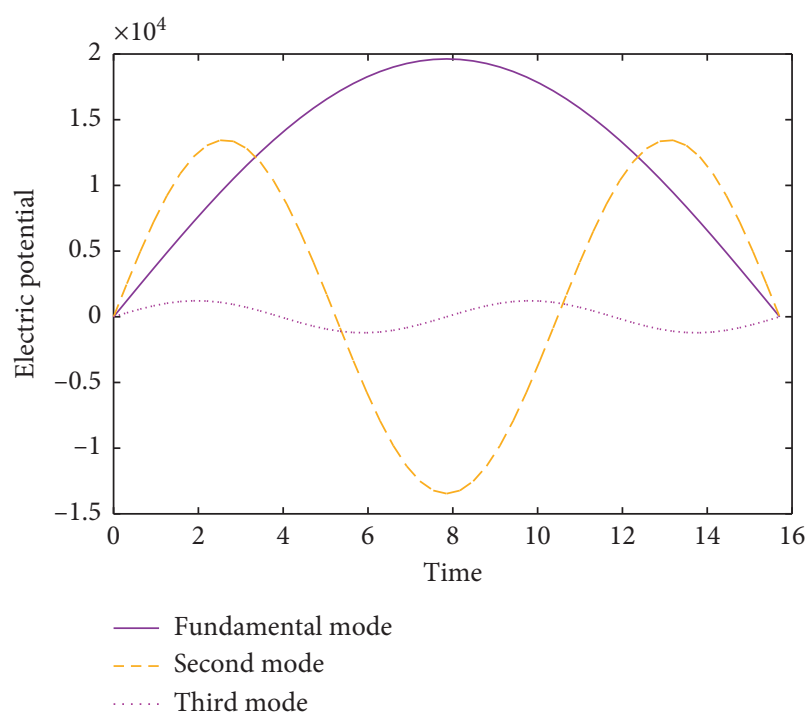

(b)

FIgURE 12: Continued. 


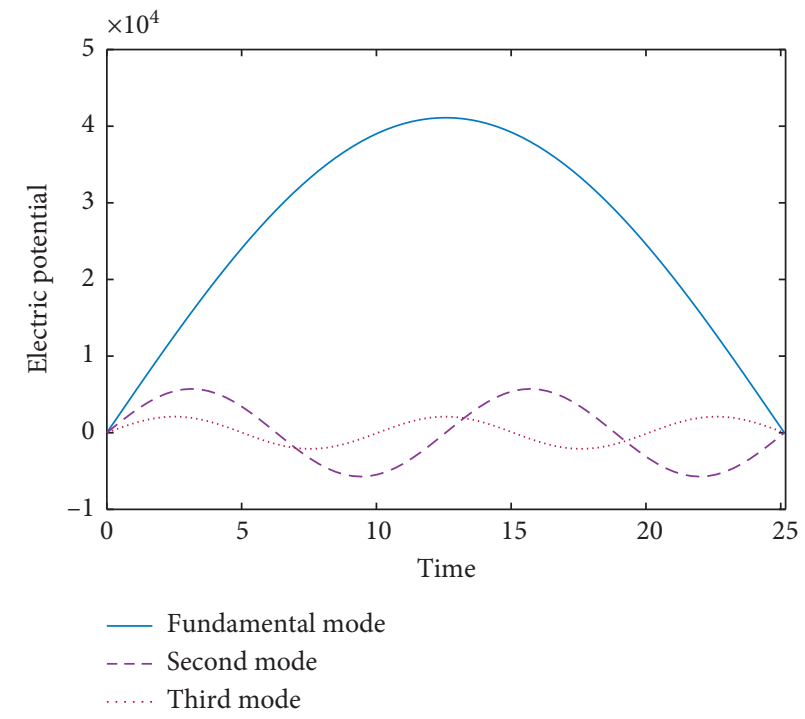

(c)

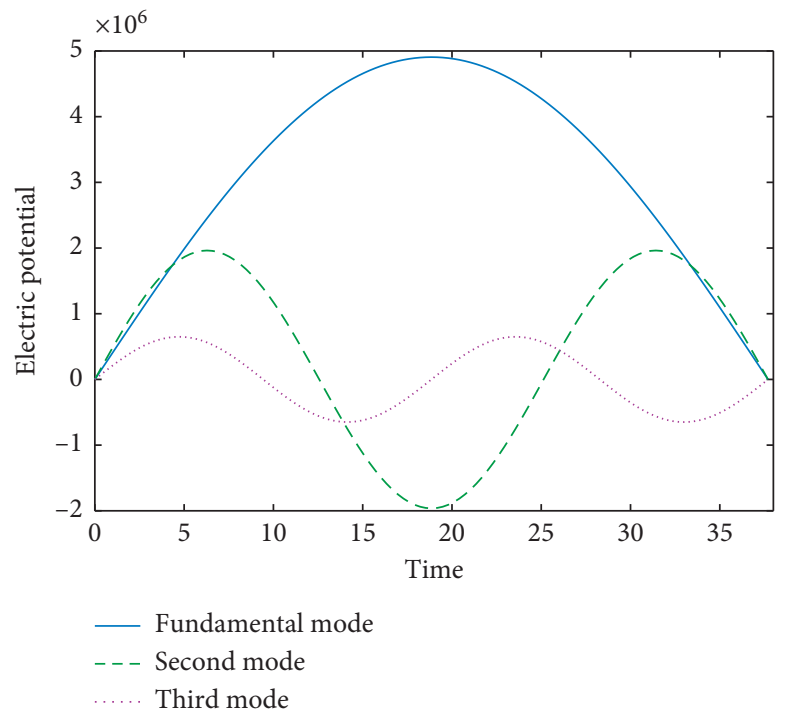

(d)

FIgURE 12: Variation of electric potential with the time for the first three modes at different boundary conditions for the square plate $\left(\overline{K_{1}}=1000, \overline{K_{2}}=100, \overline{K_{3}}=100, h_{c} / h_{p}=25, E_{1} / E_{2}=25\right.$, and $\left.a / h=10\right)$. (a) SSSS, (b) CCCC, (c) SCSC, and (d) CCCF.

\section{Conclusions}

Accurate quadrature schemes have been applied for free vibration analysis of piezoelectric composite plates with and without nonlinear elastic foundations for different types of boundary conditions. Numerical results were given to demonstrate the convergence, accuracy, and efficiency of the present methods. A MATLAB program was designed for each one, such that the maximum error (comparing with the previous exact results) was $\leq 10^{-8}$. Additionally, the execution time for each scheme was determined. It is concluded that the convergence speed for the discrete singular convolution differential quadrature method based on regularized Shannon kernel (DSCDQM-RSK) with grid size $\geq 5 \times 5 \times 5$, bandwidth $2 M+1 \geq 3$, and regularization parameter $\sigma=1.9 h_{x}$ is very fast and has the best accurate efficient results for the concerned problem (three-layered piezoelectric composite with total thickness $=0.075$ in the CCCC plate and $h=0.035$ in the SSSS plate). The efficiency of the iterative quadrature method is better than the perturbation technique. The influences of linear and nonlinear elastic foundation on the natural frequency and higher modes of frequency are investigated. The effect of the Pasternak parameter is larger than the effect of the linear and nonlinear Winkler coefficients on the natural frequencies. The natural frequency does not change when varying the nonlinear Winkler parameter. Additionally, the natural frequency converges at a larger value of the linear Winkler parameter. A parametric study is also introduced to investigate the influence of elastic and geometric characteristics of the composite and types of materials of the vibrated plate on the results. The nondimensional frequency for the material type $\mathrm{BaTiO}_{3}$ is larger than that for the PZT-4 material. Further, the natural frequency decreases with the decrease in the thickness of the piezo material or with the increase in the composite thickness until the value of ratio $h_{c}$ l $h_{p}$ closes to 10 , so the natural frequency remains constant. The thinner composite has frequencies larger than thick composite. The composite plate with CCCC conditions has the minimum normalized transverse displacement, whereas it has the maximum value for CCCF conditions. Additionally, the electric potential is the maximum for the CCCF plate and has a minimum value for the CSCS plate. Moreover, the vertical displacement increases slightly when enlarging the nonlinear parameter. Finally, the aim is for these results to be useful for modern engineering smart structures.

\section{Data Availability}

The data in this paper are all open, and the data used to support the findings of this study are available from the corresponding author upon request.

\section{Conflicts of Interest}

The authors declare that they have no conflicts of interest.

\section{References}

[1] P. L. Pasternak, "On A new method of analysis of an elastic foundation by means of two foundation constants," in Cosudarstrennoe Izdatelstvo Literaturi Po Stroitelstvu I Arkhitekture, Moscow, Russia, 1954.

[2] Y. Xiang, C. M. Wang, and S. Kitipornchai, "Exact vibration solution for initially stressed Mindlin plates on Pasternak foundations," International Journal of Mechanical Sciences, vol. 36, no. 4, pp. 311-316, 1994.

[3] H.-S. Shen, "Postbuckling analysis of composite laminated plates on two-parameter elastic foundations," International Journal of Mechanical Sciences, vol. 37, no. 12, pp. 1307-1316, 1995. 
[4] Y. Kiani, S. Taheri, and M. R. Eslami, "Thermal buckling of piezoelectric functionally graded material beams," Journal of Thermal Stresses, vol. 34, no. 8, pp. 835-850, 2011.

[5] A. Kargani, Y. Kiani, and M. R. Eslami, "Exact solution for nonlinear stability of piezoelectric FGM timoshenko beams under thermo-electrical loads," Journal of Thermal Stresses, vol. 36, no. 10, pp. 1056-1076, 2013.

[6] M. Komijani, Y. Kiani, and M. R. Eslami, "Non-linear thermoelectrical stability analysis of functionally graded piezoelectric material beams," Journal of Intelligent Material Systems and Structures, vol. 24, no. 4, pp. 399-410, 2013.

[7] Y. Kiani, "Free vibration of functionally graded carbon nanotube reinforced composite plates integrated with piezoelectric layers," Computers \& Mathematics with Applications, vol. 72, no. 9, pp. 2433-2449, 2016.

[8] Y. Kiani, M. Sadighi, and M. R. Eslami, "Dynamic analysis and active control of smart doubly curved FGM panels," Composite Structures, vol. 102, pp. 205-216, 2013.

[9] S. Pengcheng and H. Peixiang, "Analysis of bending, vibration and stability for thin plate on elastic foundation by the multivariable spline element method," Applied Mathematics and Mechanics, vol. 18, no. 8, pp. 779-787, 1997.

[10] S.-q. Li and H. Yuan, "Quasi-Green's function method for free vibration of clamped thin plates on Winkler foundation," Applied Mathematics and Mechanics, vol. 32, no. 3, pp. 265-276, 2011.

[11] S. Hatami, M. Azhari, and M. Saadatpour, "Stability and vibration of elastically supported, axially moving orthotropic plates," Iranian Journal of Science and Technology, vol. 30, pp. 427-446, 2006.

[12] S. Hosseini-Hashemi, G. Heydar Roohi, and D. T. Hossein Rokni, "Exact free vibration study of rectangular Mindlin plates with all-over part-through open cracks," Computers \& Structures, vol. 88, no. 17-18, pp. 1015-1032, 2010.

[13] L. A. Bergman, J. K. Hall, G. G. G. Lueschen, and D. M. McFarland, "Dynamic green's functions for Levy plates," Journal of Sound and Vibration, vol. 162, no. 2, pp. 281-310, 1993.

[14] K. Y. Lam, C. M. Wang, and X. Q. He, "Canonical exact solutions for Levy-plates on two-parameter foundation using Green's functions," Engineering Structures, vol. 22, no. 4, pp. 364-378, 2000.

[15] O. J. Svec, “Thick plates on elastic foundation by finite elements," Journal of the Engineering Mechanics Division, vol. 102, pp. 461-477, 1976.

[16] Q. H. Qin, "Hybrid Trefftz finite-element approach for plate bending on an elastic foundation," Applied Mathematical Modelling, vol. 18, no. 6, pp. 334-339, 1994.

[17] Q. H. Qin, "Hybrid-Trefftz finite element method for Reissner plates on an elastic foundation," Computer Methods in Applied Mechanics and Engineering, vol. 122, no. 3-4, pp. 379392, 1995.

[18] J. Costa and C. A. Brebbia, "The boundary element method applied to plates on elastic foundations," Engineering Analysis with Boundary Elements, vol. 2, no. 4, pp. 174-183, 1985.

[19] J.-B. Han and K. M. Liew, "Numerical differential quadrature method for Reissner/Mindlin plates on two-parameter foundations," International Journal of Mechanical Sciences, vol. 39, no. 9, pp. 977-989, 1997.

[20] Z. Y. Huang, C. F. Lü, and W. Q. Chen, "Benchmark solutions for functionally graded thick plates resting on Winkler-Pasternak elastic foundations," Composite Structures, vol. 85, no. 2, pp. 95-104, 2008.
[21] J. Ying, C. F. Lü, and W. Q. Chen, "Two-dimensional elasticity solutions for functionally graded beams resting on elastic foundations," Composite Structures, vol. 84, no. 3, pp. 209219, 2008.

[22] J. E. Ashton and M. E. Waddoups, "Analysis of anisotropic plates," Journal of Composite Materials, vol. 3, no. 1, pp. 148-165, 1969.

[23] J. E. Ashton, "Anisotropic plate analysis-boundary conditions," Journal of Composite Materials, vol. 4, no. 2, pp. 162-171, 1970.

[24] R. M. Jones, Mechanics of Composite Materials, Hemisphere Publishing, New York, NY, USA, 1975.

[25] M. A. Stone and H. D. Chandler, "Errors in double sine series solutions for simply supported symmetrically laminated plates," International Journal of Mechanical Sciences, vol. 38, no. 5, pp. 517-526, 1996.

[26] Q. S. Li and Y. Q. Huang, "Moving least-squares differential quadrature method for free vibration of antisymmetric laminates," Journal of Engineering Mechanics, vol. 130, no. 12, pp. 1447-1457, 2004.

[27] N. Bellomo, E. De Angelis, L. Graziano, and A. Romano, "Solution of nonlinear problems in applied sciences by generalized collocation methods and mathematica," Computers \& Mathematics with Applications, vol. 41, no. 10-11, pp. 1343-1363, 2001.

[28] M. El-Gamel, S. H. Behiry, and H. Hashish, "Numerical method for the solution of special nonlinear fourth-order boundary value problems," Applied Mathematics and Computation, vol. 145, no. 2-3, pp. 717-734, 2003.

[29] J. D. Dockery, "Numerical solution of travelling waves for reaction-diffusion equations via the sinc-galerkin method," in Proceedings of the Computation and Control, pp. 95-113, Bozeman, MT, USA, 1992.

[30] M. El-Gamel and A. Zayed, "A Comparison between the wavelet galerkin and the sincgalerkin methods in solving non homogeneous heat equations," in Series, Inverse Problems, Image Analysis, and Medical Imaging, N. Zuhair and S. Otmar, Eds., AMS, Providence, RI, USA, 2002.

[31] G. Y. Yin, "Sinc-collocation method with orthogonalization for singular Poisson-like problems," Mathematics of Computation, vol. 62, no. 205, p. 21, 1994.

[32] T. S. Carlson, J. Dockery, and J. Lund, "A sinc-collocation method for initial value problems," Mathematics of Computation, vol. 66, no. 217, pp. 215-236, 1997.

[33] C. H. W. Ng, Y. B. Zhao, and G. W. Wei, "Comparison of discrete singular convolution and generalized differential quadrature for the vibration analysis of rectangular plates," Computer Methods in Applied Mechanics and Engineering, vol. 193, no. 23-26, pp. 2483-2506, 2004.

[34] O. Ragb, M. Salah, M. S. Matbuly, and R. M. Amer, "Vibration analysis of piezoelectric composite using sinc and discrete singular convolution differential quadrature techniques," Journal of Engineering and Applied Sciences, vol. 14, no. 17, pp. 6540-6553, 2019.

[35] G. W. Wei, "A unified method for solving Maxwell's equation," in Proceedings of the Asia Pacific Microwave Conference, pp. 562-565, Singapore, 1999.

[36] G. W. Wei, "Solving quantum eigenvalue problems by discrete singular convolution," Journal of Physics B: Atomic, Molecular and Optical Physics, vol. 33, no. 3, pp. 343-352, 2000.

[37] G. W. Wei, "A unified approach for the solution of the Fokker-Planck equation," Journal of Physics A: Mathematical and General, vol. 33, no. 27, pp. 4935-4953, 2000. 
[38] G. W. Wei, Y. B. Zhao, and Y. Xiang, "A novel approach for the analysis of high-frequency vibrations," Journal of Sound and Vibration, vol. 257, no. 2, pp. 207-246, 2002.

[39] G. W. Wei, "A new algorithm for solving some mechanical problems," Computer Methods in Applied Mechanics and Engineering, vol. 190, no. 15-17, pp. 2017-2030, 2001.

[40] D. C. Wan, B. S. V. Patnaik, and G. W. Wei, "Discrete singular convolution-finite subdomain method for the solution of incompressible viscous flows," Journal of Computational Physics, vol. 180, no. 1, pp. 229-255, 2002.

[41] G. W. Wei, Y. B. Zhao, and Y. Xiang, "The determination of natural frequencies of rectangular plates with mixed boundary conditions by discrete singular convolution," International Journal of Mechanical Sciences, vol. 43, no. 8, pp. 1731-1746, 2001.

[42] G. W. Wei, "Discrete singular convolution for the solution of the Fokker-Planck equation," The Journal of Chemical Physics, vol. 110 , no. 18 , pp. 8930-8942, 1999.

[43] J. S. Nadjafi and A. Ghorbani, "He's homotopy perturbation method: an effective tool for solving nonlinear integral and integro-differential equations," Computers \& Mathematics with Applications, vol. 58, pp. 2379-2390, 2009.

[44] O. Ragb, L. F. Seddek, and M. S. Matbuly, "Iterative differential quadrature solutions for Bratu problem," Computers \& Mathematics with Applications, vol. 74, no. 2, pp. 249-257, 2017.

[45] O. Ragb, M. Mohamed, and M. S. Matbuly, "Free vibration of a piezoelectric nanobeam resting on nonlinear WinklerPasternak foundation by quadrature methods," Heliyon, vol. 5, Article ID e01856, 2019.

[46] O. Ragb, M. Mohamed, and M. S. Matbuly, "Vibration analysis of magneto-electro-thermo NanoBeam resting on nonlinear elastic foundation using sinc and discrete singular convolution differential quadrature method," Modern Applied Science, vol. 13, no. 7, p. 49, 2019.

[47] B. Akgöz and O. Civalek, "Nonlinear vibration analysis of laminated plates resting on nonlinear two-parameters elastic foundations," Steel \& Composite Structures, vol. 11, no. 5, pp. 403-421, 2011.

[48] A. M. Zenkour, "A quasi-3D refined theory for functionally graded single-layered and sandwich plates with porosities," Composite Structures, vol. 201, pp. 38-48, 2018.

[49] Ö. Civalek and M. H. Acar, "Discrete singular convolution method for the analysis of Mindlin plates on elastic foundations," International Journal of Pressure Vessels and Piping, vol. 84, no. 9, pp. 527-535, 2007.

[50] O. Civalek, "Linear vibration analysis of isotropic conical shells by discrete singular convolution (DSC)," Structural Engineering and Mechanics, vol. 25, no. 1, pp. 127-130, 2007.

[51] E. Bagherizadeh, Y. Kiani, and M. R. Eslami, "Mechanical buckling of functionally graded material cylindrical shells surrounded by Pasternak elastic foundation," Composite Structures, vol. 93, no. 11, pp. 3063-3071, 2011.

[52] O. Civalek, "Vibration analysis of conical panels using the method of discrete singular convolution," Communications in Numerical Methods in Engineering, vol. 24, no. 3, pp. 169-181, 2006.

[53] A. M. Zenkour, "Trigonometric solution for an exponentially graded thick plate resting on elastic foundations," Archive of Applied Mechanics, vol. 65, pp. 193-208, 2018.

[54] O. Civalek, "Free vibration analysis of composite conical shells using the discrete singular convolution algorithm," Steel and Composite Structures, vol. 6, no. 4, pp. 353-366, 2006.
[55] Ö. Civalek, "Fundamental frequency of isotropic and orthotropic rectangular plates with linearly varying thickness by discrete singular convolution method," Applied Mathematical Modelling, vol. 33, no. 10, pp. 3825-3835, 2009.

[56] I. Senjanovi, M. Tomi, N. Vladimir, and D. S. Cho, "Analytical solution for free vibrations of a moderately thick rectangular plate," Mathematical Problems in Engineering, vol. 2013, Article ID 207460, 13 pages, 2013.

[57] Ö. Civalek, "Free vibration and buckling analyses of composite plates with straight-sided quadrilateral domain based on DSC approach," Finite Elements in Analysis and Design, vol. 43, no. 13, pp. 1013-1022, 2007.

[58] J. Awrejcewicz, V. A. Krysko, and I. V. Kravtsova, "Dynamics and statics of flexible axially symmetric shallow shells," Mathematical Problems in Engineering, vol. 2006, Article ID 71548, 25 pages, 2006.

[59] M. Feri, A. Alibeigloo, and A. A. Pasha Zanoosi, “Three dimensional static and free vibration analysis of cross-ply laminated plate bonded with piezoelectric layers using differential quadrature method," Meccanica, vol. 51, no. 4, pp. 921-937, 2015.

[60] H. Ait Atmane, A. Tounsi, I. Mechab, and E. A. Adda Bedia, "Free vibration analysis of functionally graded plates resting on Winkler-Pasternak elastic foundations using a new shear deformation theory," International Journal of Mechanics and Materials in Design, vol. 6, no. 2, pp. 113-121, 2010.

[61] A. Hasani Baferani, A. R. Saidi, and H. Ehteshami, "Accurate solution for free vibration analysis of functionally graded thick rectangular plates resting on elastic foundation," Composite Structures, vol. 93, no. 7, pp. 1842-1853, 2011.

[62] M. e. S. Sari and W. G. Al-Kouz, "Vibration analysis of nonuniform orthotropic Kirchhoff plates resting on elastic foundation based on nonlocal elasticity theory," International Journal of Mechanical Sciences, vol. 114, pp. 1-11, 2016.

[63] N. D. Duc and P. H. Cong, "Nonlinear thermomechanical dynamic analysis and vibration of higher order shear deformable piezoelectric functionally graded material sandwich plates resting on elastic foundations," Journal of Sandwich Structures and Materials, vol. 20, pp. 191-218, 2018.

[64] Ö. Civalek, "Nonlinear analysis of thin rectangular plates on Winkler-Pasternak elastic foundations by DSC-HDQ methods," Applied Mathematical Modelling, vol. 31, no. 3, pp. 606-624, 2007.

[65] K.-J. Bathe and E. L. Wilson, "Solution methods for eigenvalue problems in structural mechanics," International Journal for Numerical Methods in Engineering, vol. 6, no. 2, pp. 213-226, 1973.

[66] Y. Y. Zhou, W. Q. Chen, and C. F. Lü, "Semi-analytical solution for orthotropic piezoelectric laminates in cylindrical bending with interfacial imperfections," Composite Structures, vol. 92, no. 4, pp. 1009-1018, 2010.

[67] A. Alibeigloo, "Three-dimensional exact solution for functionally graded rectangular plate with integrated surface piezoelectric layers resting on elastic foundation," Mechanics of Advanced Materials and Structures, vol. 17, no. 3, pp. 183-195, 2010.

[68] H. Akhavan, S. H. Hashemi, H. R. D. Taher, A. Alibeigloo, and S. Vahabi, "Exact solutions for rectangular Mindlin plates under in-plane loads resting on Pasternak elastic foundation. Part II: frequency analysis," Computational Materials Science, vol. 44, no. 3, pp. 951-961, 2009.

[69] K. Nedri, N. El Meiche, and A. Tounsi, "Free vibration analysis of laminated composite plates resting on elastic foundations by using a refined hyperbolic shear deformation theory," Mechanics of Composite Materials, vol. 49, no. 6, pp. 629-640, 2014. 